\title{
Distilling topological entropy from a single measurement of entanglement on projected systems
}

\author{
C. Castelnovo ${ }^{1}$ \\ ${ }^{1}$ TCM group, Cavendish Laboratory, University of Cambridge, Cambridge CB3 OHE, United Kingdom
}

(Dated: March 17, 2014)

\begin{abstract}
Entanglement measures find frequent application in the study of topologically ordered systems, where the presence of topological order is reflected in an additional contribution to the entanglement of the system. Obtaining this topological entropy from analytical calculations or numerical simulations is generally difficult due to the fact that it is an order one correction to leading terms that scale with the size of the system. In order to distil the topological entropy, one resorts to extrapolation as a function of system size, or to clever subtraction schemes that allow to cancel out the leading terms. Both approaches have the disadvantage of requiring multiple (accurate) calculations of the entanglement of the system. Here we propose a modification of conventional entanglement calculations that allows to obtain the topological entropy of a system from a single measurement of entanglement. In our approach, we replace the conventional trace over the degrees of freedom of a partition of the system with a projection onto a given state (which needs not be known). We show that a proper choice of partition and projective measurement allows to rid the entanglement measures of the typical boundary terms, thus exposing the topological contribution alone. We consider specifically the measures known as von Neumann entropy and entanglement negativity, and we discuss their application to both models that exhibit quantum as well as classical topological order.
\end{abstract}

\section{INTRODUCTION}

Entanglement measures, such as the von Neumann entropy or the entanglement negativity, are often used to study and characterise topologically ordered systems. It was indeed demonstrated that the presence of topological order gives rise to an additional contribution to the entanglement of the system, which relates directly to the quantum dimension of its anyonic excitations $[1,2]$. This contribution was dubbed topological entanglement entropy.

Obtaining the topological entropy of a system from analytical calculations or numerical simulations is in general a tall order. This is due to the fact that the desired contribution is an order one correction to leading terms that scale with the size of the system. For example, the von Neumann entropy of a bipartition $\mathcal{S}=\mathcal{A} \cup \mathcal{B}$ is known to exhibit an area law contribution that scales with the size of the boundary between $\mathcal{A}$ and $\mathcal{B}$. The topological entanglement entropy $\gamma$ is an order one correction to it. It was recently demonstrated that the entanglement negativity [3-7] behaves in a similar way [8,9].

In order to distil the topological entropy, one has to resort either to extrapolations as a function of system size (see e.g., Ref. 10) or to clever subtraction schemes that allow to cancel out the leading terms and to expose the order one topological correction [1,2]. Both approaches have the disadvantage of requiring multiple (and $\mathcal{O}(1)$ accurate) calculations of the entanglement of the system.

Here we propose a modification of conventional entanglement calculations that allows us to obtain the topological entropy of a system from a single measurement of entanglement. The key feature in our approach is to replace the conventional trace over the degrees of freedom of a partition of the system with a projection onto a given state (which needs not be known),

$$
\rho_{\mathcal{A}} \propto\left\langle\phi_{\mathcal{B}}|\rho| \phi_{\mathcal{B}}\right\rangle \quad \text { vs. } \quad \rho_{\mathcal{A}} \propto \operatorname{Tr}_{\mathcal{B}} \rho .
$$

We show that a proper choice of partitions and projective measurement allows to rid the entanglement measures of the typ- ical boundary terms, thus exposing the topological contribution alone.

We consider specifically the measures known as von Neumann entropy and entanglement negativity, and we discuss their application to models that exhibit quantum as well as classical topological order. The models of choice will be Kitaev's toric code model and the eight vertex model, for they allow an exact calculation of both the von Neumann entropy and the entanglement negativity.

If the von Neumann entanglement entropy is used, the resulting topological contribution can be either due to classical or quantum topological correlations. For instance, the result is the same for the classical eight-vertex model as for the quantum toric code. On the other hand, the entanglement negativity is sensitive only to quantum topological correlations and gives a non-vanishing result only in the case of quantum topological order, as in Kitaev's toric code. Therefore, the combined use of von Neumann and negativity calculations allows a straightforward detection of topological order in both classical and quantum systems, as well as a clear distinction between the two cases.

\section{TORIC CODE AND MEASURES OF ENTANGLEMENT}

The toric code is a system of spin- $1 / 2$ degrees of freedom $\sigma_{i}$ living on the bonds $i$ of a square lattice (periodic boundary conditions are assumed throughout). The Hamiltonian of the system can be written as [11]:

$$
\begin{gathered}
H=-\lambda_{A} \sum_{s} A_{s}-\lambda_{B} \sum_{p} B_{p} \\
A_{s}=\prod_{i \in s} \sigma_{i}^{x} \quad B_{p}=\prod_{i \in p} \sigma_{i}^{z},
\end{gathered}
$$

where $s(p)$ label the sites (plaquettes) of the lattice.

The ground state (GS) is 4-fold degenerate, according to the 4 topological sectors identified by the expectation values 
of winding loop operators. Within each sector, the GS is given by the equal amplitude superposition of all tensor product basis states $\otimes_{i}\left|\sigma_{i}^{z}\right\rangle$ belonging to that sector. Following the notation in Refs. 12 and 13, we introduce the group $G$ generated by products of $A_{s}$ operators. Notice that one has to define elements $g \in G$ modulo the identity $\prod_{s} A_{s}=\mathbb{I}$ in order for the inverse of $g$ to be uniquely defined (in which case, $g^{-1}=g$ ). The order (i.e., the number of elements) of $G$ is therefore $|G|=2^{N^{(s)}-1}$, where $N^{(s)}$ is the number of sites on the lattice. If we define $|0\rangle \equiv \otimes_{i}\left|\sigma_{i}^{z}=+1\right\rangle$, one of the 4 topologically ordered GS wavefunctions can be written explicitly as:

$$
\left|\psi_{0}\right\rangle=\frac{1}{|G|^{1 / 2}} \sum_{g \in G} g|0\rangle
$$

Note that the choice of reference state $|0\rangle$ is immaterial and one can replace $|0\rangle$ with $\bar{g}|0\rangle$, for any given $\bar{g} \in G$, and the state $\left|\psi_{0}\right\rangle$ remains unchanged. The other 3 GS wavefunctions are obtained upon choosing reference states $|0\rangle$ that are in different topological sectors with respect to $\otimes_{i}\left|\sigma_{i}^{z}=+1\right\rangle$.

The von Neumann entropy $S_{\mathrm{vN}}^{(\mathcal{A})}$ obtained for a bipartition of the system $\mathcal{S}=\mathcal{A} \cup \mathcal{B}$ is defined as $S_{\mathrm{vN}}^{(\mathcal{A})}=-\operatorname{Tr} \rho_{\mathcal{A}} \ln \rho_{\mathcal{A}}$, where $\rho_{\mathcal{A}}=\operatorname{Tr}_{\mathcal{B}} \rho$.

The negativity $\mathcal{N}$ (or, equivalently, the logarithmic negativity $\mathcal{E}$ ), is defined from the trace norm $\left\|\rho^{T_{\mathcal{B}}}\right\|_{1}$ of the partial transpose over subsystem $\mathcal{B}$ of the density matrix $\rho$,

$$
\mathcal{N} \equiv \frac{\left\|\rho^{T_{\mathcal{B}}}\right\|_{1}-1}{2}, \quad \mathcal{E} \equiv \ln \left\|\rho^{T_{\mathcal{B}}}\right\|_{1}
$$

where $\left\|\rho^{T_{\mathcal{B}}}\right\|_{1}$ is the sum of the absolute values of the eigenvalues $\lambda_{i}$ of $\rho^{T_{\mathcal{B}}}$. If all the eigenvalues are positive then $\mathcal{N}=0$ (recall that $\sum_{i} \lambda_{i}=1$ ) and $\mathcal{N}>0$ otherwise.

In the following we use the conventional replica trick to calculate the von Neumann entropy (see e.g., Refs. 12-14). We also use a replica approach to compute the negativity of the system which was recently introduced by Calabrese, Cardy and Tonni [15] (see also Refs. 16 and 17). This replica approach has already been applied to the toric code model in Refs. 8 and 9.

\section{PARTITION AND PROJECTION}

The method proposed in this paper requires the use of bipartitions of a system $\mathcal{S}=\mathcal{A} \cup \mathcal{B}$, where subsystem $\mathcal{B}$ splits $\mathcal{A}$ into two disconnected components, $\mathcal{A}=\mathcal{A}_{1} \cup \mathcal{A}_{2}$, and vice versa, as illustrated in Fig. 1

Without loss of generality, we will assume that the toric code has been prepared in the GS $\left|\psi_{0}\right\rangle$ in Eq. (2). Earlier results for the von Neumann entropy $[12,13]$ can be straight-
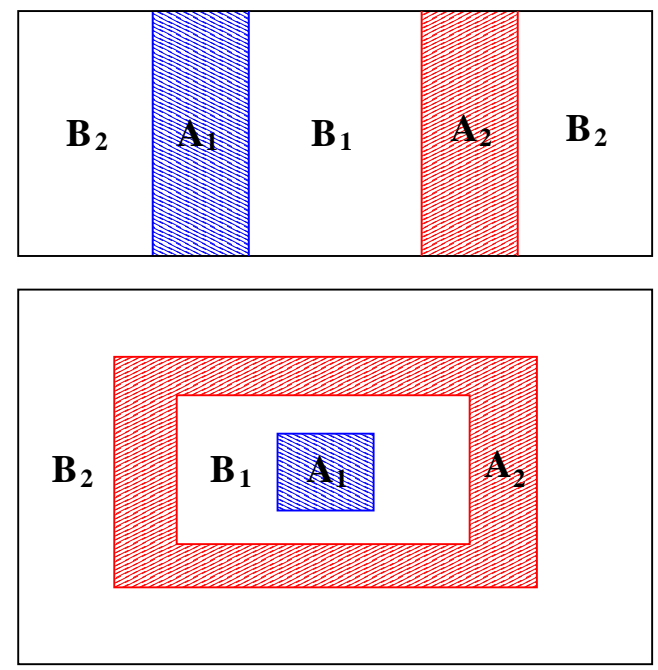

FIG. 1. (Colour online) - Examples of partitions of the system $\mathcal{S}=$ $\mathcal{A} \cup \mathcal{B}$, where subsystem $\mathcal{B}$ splits $\mathcal{A}$ into two components, $\mathcal{A}=$ $\mathcal{A}_{1} \cup \mathcal{A}_{2}$, and vice versa.

forwardly applied to show that

$$
\begin{aligned}
S_{\mathrm{vN}}^{(\mathcal{A})} & =-\operatorname{Tr} \rho_{\mathcal{A}} \ln \rho_{\mathcal{A}}=-\ln \frac{\left|G_{\mathcal{A}}\right|\left|G_{\mathcal{B}}\right|}{|G|} \\
& =\ln \left[2^{N_{\partial_{\mathcal{A}}}^{(s)}+1-\left(m_{\mathcal{A}}+m_{\mathcal{B}}\right)}\right] \\
S_{\mathrm{vN}}^{\left(\mathcal{A}_{1}\right)} & =-\operatorname{Tr} \rho_{\mathcal{A}_{1}} \ln \rho_{\mathcal{A}_{1}}=-\ln \frac{\left|G_{\mathcal{A}_{1}}\right|\left|G_{\mathcal{A}_{2} \cup \mathcal{B}}\right|}{|G|} \\
& =\ln \left[2^{\left.N_{\partial_{\mathcal{A}_{1}}^{(s)}}^{s^{(s)}}\right]},\right.
\end{aligned}
$$

where $G_{\alpha}$ is the subgroup of $G$ that acts only on spins in subsystem $\alpha\left(G_{\alpha}=\left\{g \in G \mid g=g_{\alpha} \otimes \mathbf{1}_{\alpha^{c}}\right\}, \mathcal{S}=\alpha \cup \alpha^{c}\right)$, and $\left|G_{\alpha}\right|$ is its order. $N_{\partial_{\alpha}}^{(s)} \equiv N_{\partial_{\alpha} c}^{(s)}$ is the number of star operators acting simultaneously on spins in $\alpha$ and on spins in its complementary subsystem $\alpha^{c}$. Moreover, $m_{\alpha}$ is the number of disconnected components of subsystem $\alpha$.

In order to arrive at the results above, we used the fact that $\left|G_{\mathcal{A}_{1}}\right|=2^{N_{\mathcal{A}_{1}}^{(s)}}$ and $\left|G_{\mathcal{A}_{2} \cup \mathcal{B}}\right|=2^{N_{\mathcal{B}_{1}}^{(s)}+N_{\mathcal{B}_{2}}^{(s)}+N_{\mathcal{A}_{2}}^{(s)}+N_{\partial \mathcal{A}_{2}}^{(s)}}$ (both subsystems are topologically trivial), whereas $\left|G_{\mathcal{A}}\right|=$ $2^{N_{\mathcal{A}_{1}}^{(s)}+N_{\mathcal{A}_{2}}^{(s)}+m_{\mathcal{B}}-1}$ and $\left|G_{\mathcal{B}}\right|=2^{N_{\mathcal{B}_{1}}^{(s)}+N_{\mathcal{B}_{2}}^{(s)}+m_{\mathcal{A}}-1}$ (both subsystems are topologically non-trivial). The topological nature of the system is reflected in the appearance of the contributions $m_{\mathcal{A}}=2$ and $m_{\mathcal{B}}=2$, which depend solely on the topology of the partition, as we explain hereafter.

The factor $2^{m_{\alpha c-1}}$ in $\left|G_{\alpha}\right|$ is due to the fact that, if $\alpha^{c}$ has more than one disconnected component, then the product of all star operators in each component $\alpha_{i}^{c}$ times the product of all star operators of its boundary $\partial_{\alpha_{i}^{c}}$ is an operation acting solely on $\alpha$ that cannot be written in terms of star operators in $\alpha$. This is perhaps best illustrated by looking at the example in Fig. 1, top panel. Consider the product of all star operators acting solely on $\mathcal{A}_{1}$ times the product of all star operators at its boundary, i.e., acting simultaneously on spins in $\mathcal{A}_{1}$ and in $\mathcal{B}$. The resulting operators acts twice on each and 
every spin in $\mathcal{A}_{1}$, thus leaving it unchanged; it acts however on some spins in $\mathcal{B}$, and this action cannot be written in terms of products of star operators acting on $\mathcal{B}$ alone. Therefore, it is an additional operation in $G_{\mathcal{B}}$ independent from the $2^{N_{\mathcal{B}}^{(s)}}$ that one can straightforwardly construct from star operators acting only on $\mathcal{B}$. This happens because $m_{\mathcal{A}}>1$ : if $m_{\mathcal{A}}=1$ then the operation described above is in fact equivalent to the product of all star operators acting only on $\mathcal{B}$, hence the subtraction " -1 " in the exponent of the factor $2^{m_{\mathcal{A}}-1}$ appearing in $\left|G_{\mathcal{B}}\right|$.

Whichever the choice to calculate the von Neumann entropy, the topological contribution (of order one) is subordinate to a boundary term $N_{\partial_{\alpha}}^{(s)}$ that scales with the size of the partition.

Similar results were obtained recently for the entanglement negativity $[8,9]$. If one considers $\rho_{\mathcal{A}}=\operatorname{Tr}_{\mathcal{B}} \rho$ and then computes the trace norm $\left\|\rho^{T_{\mathcal{A}_{2}}}\right\|_{1}$ (after transposing the degrees of freedom in $\mathcal{A}_{2}$ ), the choice of partitions in Fig. 1 actually leads to $\left\|\rho^{T_{\mathcal{A}_{2}}}\right\|_{1}=1$ and vanishing negativity (see Refs. 8 and 9, or App. $\mathrm{D}$ for an alternative derivation). It is only when the partitions $\mathcal{A}_{1}$ and $\mathcal{A}_{2}$ share a boundary and $\mathcal{A}$ spans the system in both directions that one can see a topological contribution in the entanglement negativity. However, this is once again an order one correction to a boundary contribution that scales with the size of the partition.

In order to remove the boundary contribution without throwing the topological baby with the bath water, we consider here the density matrix of subsystem $\mathcal{A}$ after performing a projective measurement on the spins in subsystem $\mathcal{B}$ :

$$
\rho_{\mathcal{A}} \propto\left\langle\phi_{\mathcal{B}}|\rho| \phi_{\mathcal{B}}\right\rangle,
$$

where $\left|\phi_{\mathcal{B}}\right\rangle$ is taken to be a generic tensor product basis state $\otimes_{i \in \mathcal{B}}\left|\sigma_{i}^{z}\right\rangle$ appearing in the GS superposition in Eq. (2). Namely, $\left|\phi_{\mathcal{B}}\right\rangle=\bar{g}_{\mathcal{B}}\left|0_{\mathcal{B}}\right\rangle$, for some $\bar{g} \in G$. This is equivalent, for instance, to performing a measurement of the $\sigma^{z}$ component of each spin in $\mathcal{B}$.

The results that follow are independent of the choice of $\bar{g}$, and knowledge of the corresponding state $\left|\phi_{\mathcal{B}}\right\rangle$ is immaterial. Therefore, we only need to know that the spins in $\mathcal{B}$ have been measured, but we do not need to know the result of that measurement.

Given that the toric code is symmetric upon exchange of $\sigma^{z}$ and $\sigma^{x}$ operators, whilst exchanging also stars with plaquettes (mapping the direct lattice to the face-centred dual lattice), the results hold true also if the projective measurement is done on the $\sigma^{x}$ component of the spins in $\mathcal{B}$. The results however do not generically apply if $\left|\phi_{\mathcal{B}}\right\rangle$ is a superposition of tensor product states in the $\sigma^{z}$ basis. A counter example and related discussion of the conditions under which a superposition is admissible are given in $\mathrm{Sec}$. III C.

With the choice $\left|\phi_{\mathcal{B}}\right\rangle=\bar{g}_{\mathcal{B}}\left|0_{\mathcal{B}}\right\rangle$, for some $\bar{g} \in G$, after a few lines of algebra reported in App. A for convenience, one arrives at the expression

$$
\rho_{\mathcal{A}}=\frac{1}{\left|G_{\mathcal{A}}\right|} \sum_{g, g^{\prime} \in g_{\mathcal{A}}} g_{\mathcal{A}}\left|0_{\mathcal{A}}\right\rangle\left\langle 0_{\mathcal{A}}\right| g_{\mathcal{A}}^{\prime}
$$

where $\rho_{\mathcal{A}}$ has been normalised so that $\operatorname{Tr}\left(\rho_{\mathcal{A}}\right)=1$. The state $|0\rangle$ in the expression above is determined (in part) by the choice of $\left|\phi_{\mathcal{B}}\right\rangle$. However, as we shall see in the following, this plays no role in the calculation of the von Neumann entropy and entanglement negativity.

\section{A. Entanglement entropy}

Instead of computing the von Neumann entropy directly from $\rho_{\mathcal{A}}$ in Eq. (9), which trivially vanishes (see App. B), let us trace out the degrees of freedom in $\mathcal{A}_{2}$,

$$
\begin{aligned}
\rho_{\mathcal{A}_{1}} & =\frac{1}{\left|G_{\mathcal{A}}\right|} \sum_{g, g^{\prime} \in G_{\mathcal{A}}} g_{\mathcal{A}_{1}}\left|0_{\mathcal{A}_{1}}\right\rangle\left\langle 0_{\mathcal{A}_{1}}\right| g_{\mathcal{A}_{1}}^{\prime} \\
& \times\left\langle 0_{\mathcal{A}_{2}}\left|g_{\mathcal{A}_{2}}^{\prime} g_{\mathcal{A}_{2}}\right| 0_{\mathcal{A}_{2}}\right\rangle .
\end{aligned}
$$

Keeping $g$ fixed, we notice that the mapping $g^{\prime} \rightarrow \tilde{g}=$ $g^{\prime} g$ is one-to-one in $G_{\mathcal{A}}$, and therefore we can re-write the summation over $g^{\prime}$ as a summation over $\tilde{g}$ upon replacing $g^{\prime}=$ $\tilde{g} g$ (recall that $g^{2}=\mathbf{1}$ for all $g \in G$ ). The expectation value in the expression above reduces then to the condition that $\tilde{g}_{\mathcal{A}_{2}}=$ $\mathbf{1}_{\mathcal{A}_{2}}$, or equivalently $\tilde{g} \in G_{\mathcal{A}_{1}}$ :

$$
\rho_{\mathcal{A}_{1}}=\frac{1}{\left|G_{\mathcal{A}}\right|} \sum_{g \in G_{\mathcal{A}}, \tilde{g} \in G_{\mathcal{A}_{1}}} g_{\mathcal{A}_{1}}\left|0_{\mathcal{A}_{1}}\right\rangle\left\langle 0_{\mathcal{A}_{1}}\right| g_{\mathcal{A}_{1}} \tilde{g}_{\mathcal{A}_{1}} .
$$

We can then compute $S_{\mathrm{vN}}^{\left(\mathcal{A}_{1}\right)}=-\operatorname{Tr}\left[\rho_{\mathcal{A}_{1}} \ln \rho_{\mathcal{A}_{1}}\right]$ using the replica trick $-\lim _{n \rightarrow 1} \partial_{n}\left[\operatorname{Tr}\left(\rho_{\mathcal{A}_{1}}^{n}\right)\right]$. In order to do so, we start by considering

$$
\begin{aligned}
\rho_{\mathcal{A}_{1}}^{2} & =\frac{1}{\left|G_{\mathcal{A}}\right|^{2}} \sum_{g, g^{\prime} \in G_{\mathcal{A}}} \sum_{\tilde{g}, \tilde{g}^{\prime} \in G_{\mathcal{A}_{1}}} g_{\mathcal{A}_{1}}\left|0_{\mathcal{A}_{1}}\right\rangle\left\langle 0_{\mathcal{A}_{1}}\right| g_{\mathcal{A}_{1}}^{\prime} \tilde{g}_{\mathcal{A}_{1}}^{\prime} \\
& \times\left\langle 0_{\mathcal{A}_{1}}\left|g_{\mathcal{A}_{1}} \tilde{g}_{\mathcal{A}_{1}} g_{\mathcal{A}_{1}}^{\prime}\right| 0_{\mathcal{A}_{1}}\right\rangle .
\end{aligned}
$$

Once again, given $g$ and $\tilde{g}$ we can use a one-to-one mapping to replace $g^{\prime} \rightarrow g^{\prime \prime}=g \tilde{g} g^{\prime}$, with $g^{\prime \prime} \in G_{\mathcal{A}}$. The expectation value reduces to $\left\langle 0_{\mathcal{A}_{1}}\left|g_{\mathcal{A}_{1}}^{\prime \prime}\right| 0_{\mathcal{A}_{1}}\right\rangle$, which imposes $g^{\prime \prime} \in G_{\mathcal{A}_{2}}$ :

$$
\begin{aligned}
\rho_{\mathcal{A}_{1}}^{2} & =\frac{1}{\left|G_{\mathcal{A}}\right|^{2}} \sum_{g \in G_{\mathcal{A}}, g^{\prime \prime} \in G_{\mathcal{A}_{2}} \tilde{g}, \tilde{g}^{\prime} \in G_{\mathcal{A}_{1}}} \sum \\
& \times g_{\mathcal{A}_{1}}\left|0_{\mathcal{A}_{1}}\right\rangle\left\langle 0_{\mathcal{A}_{1}}\right| g_{\mathcal{A}_{1}}^{\prime \prime} g_{\mathcal{A}_{1}} \tilde{g}_{\mathcal{A}_{1}} \tilde{g}_{\mathcal{A}_{1}}^{\prime} \\
& =\frac{\left|G_{\mathcal{A}_{1}}\right|\left|G_{\mathcal{A}_{2}}\right|}{\left|G_{\mathcal{A}}\right|^{2}} \sum_{g \in G_{\mathcal{A}}} \sum_{\tilde{g} \in G_{\mathcal{A}_{1}}} g_{\mathcal{A}_{1}}\left|0_{\mathcal{A}_{1}}\right\rangle\left\langle 0_{\mathcal{A}_{1}}\right| g_{\mathcal{A}_{1}} \tilde{g}_{\mathcal{A}_{1}} \\
& =\frac{\left|G_{\mathcal{A}_{1}}\right|\left|G_{\mathcal{A}_{2}}\right|}{\left|G_{\mathcal{A}}\right|} \rho_{\mathcal{A}_{1}} .
\end{aligned}
$$

Here we used the fact that $g_{\mathcal{A}_{1}}^{\prime \prime}=\mathbf{1}_{\mathcal{A}_{1}}$; the dependence on $g^{\prime \prime}$ disappears, allowing to sum over it and resulting in the factor $\left|G_{\mathcal{A}_{2}}\right|$. Moreover, the product $\tilde{g} \tilde{g}^{\prime}$ is a generic element of $G_{\mathcal{A}_{1}}$ and therefore we can trivially sum over, say, $\tilde{g}^{\prime}$, resulting in the factor $\left|G_{\mathcal{A}_{1}}\right|$.

Iterating this identity we obtain

$$
\operatorname{Tr}\left(\rho_{\mathcal{A}_{1}}^{n}\right)=\left(\frac{\left|G_{\mathcal{A}_{1}}\right|\left|G_{\mathcal{A}_{2}}\right|}{\left|G_{\mathcal{A}}\right|}\right)^{n-1}
$$

and $S_{\mathrm{vN}}^{\left(\mathcal{A}_{1}\right)}=-\ln \left(\left|G_{\mathcal{A}_{1}}\right|\left|G_{\mathcal{A}_{2}}\right| /\left|G_{\mathcal{A}}\right|\right)$. As discussed above, $\mathcal{A}_{1}$ and $\mathcal{A}_{2}$ are topologically trivial and therefore $\left|G_{\mathcal{A}_{1}}\right|=$ 
$2^{N_{\mathcal{A}_{1}}^{(s)}}$ and $\left|G_{\mathcal{A}_{2}}\right|=2^{N_{\mathcal{A}_{2}}^{(s)}}$. On the other hand, $\mathcal{A}$ divides subsystem $\mathcal{B}$ in two disconnected components, and therefore $\left|G_{\mathcal{A}}\right|=2^{N_{\mathcal{A}}^{(s)}}+m_{\mathcal{B}}-1$, with $m_{\mathcal{B}}=2$ and $N_{\mathcal{A}}^{(s)}=$ $N_{\mathcal{A}_{1}}^{(s)}+N_{\mathcal{A}_{2}}^{(s)}$. We finally obtain $S_{\mathrm{vN}}^{\left(\mathcal{A}_{1}\right)}=\ln 2$, which is indeed the expected value of the topological entropy, without any boundary contribution.

\section{B. Negativity}

Let us now consider the entanglement negativity between subsystem $\mathcal{A}_{1}$ and $\mathcal{A}_{2}$ after the projection of subsystem $\mathcal{B}$. Firstly, we take the transpose of $\rho_{\mathcal{A}}$ over the degrees of free$\operatorname{dom}$ in $\mathcal{A}_{2}$,

$$
\begin{aligned}
\rho_{\mathcal{A}}^{T_{2}} & =\frac{1}{\left|G_{\mathcal{A}}\right|} \sum_{g, g^{\prime} \in g_{\mathcal{A}}} \\
& \times\left(g_{\mathcal{A}_{1}}\left|0_{\mathcal{A}_{1}}\right\rangle\left\langle 0_{\mathcal{A}_{1}}\right| g_{\mathcal{A}_{1}}^{\prime}\right) \otimes\left(g_{\mathcal{A}_{2}}^{\prime}\left|0_{\mathcal{A}_{2}}\right\rangle\left\langle 0_{\mathcal{A}_{2}}\right| g_{\mathcal{A}_{2}}\right),
\end{aligned}
$$

and then we compute its second power,

$$
\begin{aligned}
\left(\rho_{\mathcal{A}}^{T_{2}}\right)^{2} & =\frac{1}{\left|G_{\mathcal{A}}\right|^{2}} \sum_{g, g^{\prime} \in g_{\mathcal{A}}} \sum_{\tilde{g}, \tilde{g}^{\prime} \in g_{\mathcal{A}}} \\
& \times\left(g_{\mathcal{A}_{1}}\left|0_{\mathcal{A}_{1}}\right\rangle\left\langle 0_{\mathcal{A}_{1}}\left|g_{\mathcal{A}_{1}}^{\prime} \tilde{g}_{\mathcal{A}_{1}}\right| 0_{\mathcal{A}_{1}}\right\rangle\left\langle 0_{\mathcal{A}_{1}}\right| \tilde{g}_{\mathcal{A}_{1}}^{\prime}\right) \\
& \otimes\left(g_{\mathcal{A}_{2}}^{\prime}\left|0_{\mathcal{A}_{2}}\right\rangle\left\langle 0_{\mathcal{A}_{2}}\left|g_{\mathcal{A}_{2}} \tilde{g}_{\mathcal{A}_{2}}^{\prime}\right| 0_{\mathcal{A}_{2}}\right\rangle\left\langle 0_{\mathcal{A}_{2}}\right| \tilde{g}_{\mathcal{A}_{2}}\right) .
\end{aligned}
$$

Given $g$ and $g^{\prime}$, we can change the summation variables $\tilde{g} \rightarrow \tilde{g} g^{\prime}$ and $\tilde{g}^{\prime} \rightarrow \tilde{g}^{\prime} g$ (one-to-one mapping from $G_{\mathcal{A}}$ to $G_{\mathcal{A}}$, where we re-use the same labels before and after the change of variables for notational convenience):

$$
\begin{aligned}
\left(\rho_{\mathcal{A}}^{T_{2}}\right)^{2} & =\frac{1}{\left|G_{\mathcal{A}}\right|^{2}} \sum_{g, g^{\prime} \in g_{\mathcal{A}}} \sum_{\tilde{g}, \tilde{g}^{\prime} \in g_{\mathcal{A}}} \\
& \times\left(g_{\mathcal{A}_{1}}\left|0_{\mathcal{A}_{1}}\right\rangle\left\langle 0_{\mathcal{A}_{1}}\left|\tilde{g}_{\mathcal{A}_{1}}\right| 0_{\mathcal{A}_{1}}\right\rangle\left\langle 0_{\mathcal{A}_{1}}\right| \tilde{g}_{\mathcal{A}_{1}}^{\prime} g_{\mathcal{A}_{1}}\right) \\
& \otimes\left(g_{\mathcal{A}_{2}}^{\prime}\left|0_{\mathcal{A}_{2}}\right\rangle\left\langle 0_{\mathcal{A}_{2}}\left|\tilde{g}_{\mathcal{A}_{2}}^{\prime}\right| 0_{\mathcal{A}_{2}}\right\rangle\left\langle 0_{\mathcal{A}_{2}}\right| \tilde{g}_{\mathcal{A}_{2}} g_{\mathcal{A}_{2}}^{\prime}\right) .
\end{aligned}
$$

The two expectation values can thus be seen to impose the conditions $\tilde{g}_{\mathcal{A}_{1}}=\mathbf{1}_{\mathcal{A}_{1}}$ and $\tilde{g}_{\mathcal{A}_{2}}^{\prime}=\mathbf{1}_{\mathcal{A}_{2}}$, or equivalently $\tilde{g} \in$ $G_{\mathcal{A}_{2}}$ and $\tilde{g}^{\prime} \in G_{\mathcal{A}_{1}}$ :

$$
\begin{aligned}
& \left(\rho_{\mathcal{A}}^{T_{2}}\right)^{2}=\frac{1}{\left|G_{\mathcal{A}}\right|^{2}} \sum_{g, g^{\prime} \in G_{\mathcal{A}}} \sum_{\tilde{g} \in G_{\mathcal{A}_{2}}} \sum_{\tilde{g}^{\prime} \in G_{\mathcal{A}_{1}}} \\
& \times\left(g_{\mathcal{A}_{1}}\left|0_{\mathcal{A}_{1}}\right\rangle\left\langle 0_{\mathcal{A}_{1}}\right| \tilde{g}_{\mathcal{A}_{1}}^{\prime} g_{\mathcal{A}_{1}}\right) \otimes\left(g_{\mathcal{A}_{2}}^{\prime}\left|0_{\mathcal{A}_{2}}\right\rangle\left\langle 0_{\mathcal{A}_{2}}\right| \tilde{g}_{\mathcal{A}_{2}} g_{\mathcal{A}_{2}}^{\prime}\right) .
\end{aligned}
$$

Using Eq. (17) we can proceed to compute the third power

$$
\begin{aligned}
\left(\rho_{\mathcal{A}}^{T_{2}}\right)^{3}= & \frac{1}{\left|G_{\mathcal{A}}\right|^{3}} \sum_{g, g^{\prime} \in G_{\mathcal{A}}} \sum_{\tilde{g} \in G_{\mathcal{A}_{2}}} \sum_{\tilde{g}^{\prime} \in G_{\mathcal{A}_{1}}} \sum_{h, h^{\prime} \in G_{\mathcal{A}}} \\
& \times\left(g_{\mathcal{A}_{1}}\left|0_{\mathcal{A}_{1}}\right\rangle\left\langle 0_{\mathcal{A}_{1}}\left|\tilde{g}_{\mathcal{A}_{1}}^{\prime} g_{\mathcal{A}_{1}} h_{\mathcal{A}_{1}}\right| 0_{\mathcal{A}_{1}}\right\rangle\left\langle 0_{\mathcal{A}_{1}}\right| h_{\mathcal{A}_{1}}^{\prime}\right) \\
& \otimes\left(g_{\mathcal{A}_{2}}^{\prime}\left|0_{\mathcal{A}_{2}}\right\rangle\left\langle 0_{\mathcal{A}_{2}}\left|\tilde{g}_{\mathcal{A}_{2}} g_{\mathcal{A}_{2}}^{\prime} h_{\mathcal{A}_{2}}^{\prime}\right| 0_{\mathcal{A}_{2}}\right\rangle\left\langle 0_{\mathcal{A}_{2}}\right| h_{\mathcal{A}_{2}}\right) .
\end{aligned}
$$

Given $g^{\prime} \in G_{\mathcal{A}}$ and $\tilde{g} \in G_{\mathcal{A}_{2}} \subset G_{\mathcal{A}}$, it is useful to redefine $h^{\prime} \rightarrow \tilde{g} g^{\prime} h^{\prime}$. Similarly, we can redefine $h \rightarrow \tilde{g}^{\prime} g h$. It is straightforward to show that both changes correspond to a trivial re-labelling the summation indices (one-to-one mappings of $G_{\mathcal{A}}$ onto $G_{\mathcal{A}}$ ):

$$
\begin{aligned}
\left(\rho_{\mathcal{A}}^{T_{2}}\right)^{3} & =\frac{1}{\left|G_{\mathcal{A}}\right|^{3}} \sum_{g, g^{\prime} \in G_{\mathcal{A}}} \sum_{\tilde{g} \in G_{\mathcal{A}_{2}}} \sum_{\tilde{g}^{\prime} \in G_{\mathcal{A}_{1}}} \sum_{h, h^{\prime} \in G_{\mathcal{A}}} \\
& \times\left(g_{\mathcal{A}_{1}}\left|0_{\mathcal{A}_{1}}\right\rangle\left\langle 0_{\mathcal{A}_{1}}\left|h_{\mathcal{A}_{1}}\right| 0_{\mathcal{A}_{1}}\right\rangle\left\langle 0_{\mathcal{A}_{1}}\right| \tilde{g}_{\mathcal{A}_{1}} g_{\mathcal{A}_{1}}^{\prime} h_{\mathcal{A}_{1}}^{\prime}\right) \\
& \otimes\left(g_{\mathcal{A}_{2}}^{\prime}\left|0_{\mathcal{A}_{2}}\right\rangle\left\langle 0_{\mathcal{A}_{2}}\left|h_{\mathcal{A}_{2}}^{\prime}\right| 0_{\mathcal{A}_{2}}\right\rangle\left\langle 0_{\mathcal{A}_{2}}\right| \tilde{g}_{\mathcal{A}_{2}}^{\prime} g_{\mathcal{A}_{2}} h_{\mathcal{A}_{2}}\right) .
\end{aligned}
$$

Notice that $\tilde{g}_{\mathcal{A}_{1}}=\mathbf{1}_{\mathcal{A}_{1}}$ since $\tilde{g} \in G_{\mathcal{A}_{2}}$, and $\tilde{g}_{\mathcal{A}_{2}}^{\prime}=\mathbf{1}_{\mathcal{A}_{2}}$ since $\tilde{g}^{\prime} \in G_{\mathcal{A}_{1}}$, by which the dependence on $\tilde{g}$ and $\tilde{g}^{\prime}$ disappears (and they sum to an overall factor $\left|G_{\mathcal{A}_{1}}\right|\left|G_{\mathcal{A}_{2}}\right|$ ). Moreover, we can redefine $g^{\prime} \rightarrow h^{\prime} g^{\prime}$ as well as $g \rightarrow h g$, thus transferring $h^{\prime}(h)$ from the right hand side of the second (third) line in the equation above to the left hand side of the third (second) line. The two expectation values impose the conditions $h \in G_{\mathcal{A}_{2}}$ and $h^{\prime} \in G_{\mathcal{A}_{1}}$, and therefore the dependence on $h$ and $h^{\prime}$ also disappears (producing another overall factor $\left|G_{\mathcal{A}_{1}}\right|\left|G_{\mathcal{A}_{2}}\right|$ ).

The result above thus simplifies to:

$$
\begin{aligned}
\left(\rho_{\mathcal{A}}^{T_{2}}\right)^{3} & =\frac{\left|G_{\mathcal{A}_{1}}\right|^{2}\left|G_{\mathcal{A}_{2}}\right|^{2}}{\left|G_{\mathcal{A}}\right|^{3}} \sum_{g, g^{\prime} \in G_{\mathcal{A}}} \\
& \times\left(g_{\mathcal{A}_{1}}\left|0_{\mathcal{A}_{1}}\right\rangle\left\langle 0_{\mathcal{A}_{1}}\right| g_{\mathcal{A}_{1}}^{\prime}\right) \otimes\left(g_{\mathcal{A}_{2}}^{\prime}\left|0_{\mathcal{A}_{2}}\right\rangle\left\langle 0_{\mathcal{A}_{2}}\right| g_{\mathcal{A}_{2}}\right) \\
& =\frac{\left|G_{\mathcal{A}_{1}}\right|^{2}\left|G_{\mathcal{A}_{2}}\right|^{2}}{\left|G_{\mathcal{A}}\right|^{2}} \rho_{\mathcal{A}}^{T_{2}} .
\end{aligned}
$$

where the last equality was obtained by comparison with Eq. (15). We can finally combine our results to obtain that (for $n \geq 2$ )

$$
\left(\rho_{\mathcal{A}}^{T_{2}}\right)^{n}=\left(\frac{\left|G_{\mathcal{A}_{1}}\right|^{2}\left|G_{\mathcal{A}_{2}}\right|^{2}}{\left|G_{\mathcal{A}}\right|^{2}}\right)^{k} \begin{cases}\rho_{\mathcal{A}}^{T_{2}} & \text { if } n=2 k+1 \\ \left(\rho_{\mathcal{A}}^{T_{2}}\right)^{2} & \text { if } n=2 k+2 .\end{cases}
$$

Given the fact that $\operatorname{Tr}\left(\rho_{\mathcal{A}}^{T_{2}}\right)=1$ and $\operatorname{Tr}\left[\left(\rho_{\mathcal{A}}^{T_{2}}\right)^{2}\right]=1$, then

$$
\operatorname{Tr}\left[\left(\rho_{\mathcal{A}}^{T_{2}}\right)^{n}\right]= \begin{cases}\left(\frac{\left|G_{\mathcal{A}_{1}}\right| G_{\mathcal{A}_{2}} \mid}{\left|G_{\mathcal{A}}\right|}\right)^{n-1} \text { if } n \text { is odd } \\ \left(\frac{\left|G_{\mathcal{A}_{1}}\right|\left|G_{\mathcal{A}_{2}}\right|}{\left|G_{\mathcal{A}}\right|}\right)^{n-2} \text { if } n \text { is even }\end{cases}
$$

Following the replica approach proposed in Ref. 15, we see that the analytic continuation of Eq. (20) for $n \rightarrow 1$ differs whether we follow the even or odd power sequence. The odd sequence tends to 1 , as expected for the trace of $\rho_{\mathcal{A}}^{T_{2}}$. The even sequence tends instead to the sum of the absolute values of the eigenvalues of $\rho_{\mathcal{A}}^{T_{2}}$ and it does not converge to 1 , thus signalling a non-vanishing entanglement negativity:

$$
\mathcal{E} \equiv \ln \left\|\rho_{\mathcal{A}}^{T_{2}}\right\|_{1}=\ln \frac{\left|G_{\mathcal{A}}\right|}{\left|G_{\mathcal{A}_{1}}\right|\left|G_{\mathcal{A}_{2}}\right|}=\ln 2 .
$$

Once again, we obtain a direct measure of the topological entropy of the system without any boundary term or other contribution that scales with the size of the partition. 


\section{Choice of the projected state}

It is important to stress here that the results obtained thus far (both for the von Neumann entropy as well as the entanglement negativity) do not depend in any way on the specific choice of state $\left|0_{\mathcal{A}}\right\rangle$. Therefore, they are also independent from the specific state that subsystem $\mathcal{B}$ is projected onto, provided it has a non-vanishing overlap with the ground state of the system.

This holds true under the assumption that $\left|\phi_{\mathcal{B}}\right\rangle$ is a tensor product state of the $\sigma_{i}^{z}$ operators. Given the symmetry of the toric code upon exchanging $x \leftrightarrow z$ and stars with plaquettes, similar calculations and results hold true for projections on $\sigma_{i}^{x}$ tensor product states.

What about more general projective measurements on $\mathcal{B}$ ? We can always express a generic state $\left|\phi_{\mathcal{B}}\right\rangle$ as a superposition of tensor product states in the $\sigma^{z}$ basis. The only tensor product states that are relevant to the present work are those which have non-vanishing overlap with the GS wavefunction of the toric code, i.e., of the form $\bar{g}_{\mathcal{B}}\left|0_{\mathcal{B}}\right\rangle$ for some $\bar{g} \in G$. In App. E we discuss in detail the instructive example of a superposition of two such states. Here we report a summary of the results and relative discussion. We find that one ought to distinguish between states, say $\bar{g}$ and $\overline{\bar{g}}$, according to the whether at least one of the following conditions is satisfied:

$$
\begin{aligned}
\exists h \in G_{\mathcal{A}} & : h_{\mathcal{A}_{2}} \bar{g}_{\mathcal{A}_{2}}=\overline{\bar{g}}_{\mathcal{A}_{2}} \\
\exists h^{\prime} \in G_{\mathcal{A}} & : h_{\mathcal{A}_{1}}^{\prime} \bar{g}_{\mathcal{A}_{1}}=\overline{\bar{g}}_{\mathcal{A}_{1}} .
\end{aligned}
$$

If the answer is positive, one can then use the freedom in the choice of elements of subgroup $G_{\mathcal{A}} \subset G$ (which necessarily survives after projecting out $\mathcal{B}$ ) to interchange $\bar{g}$ with $\overline{\bar{g}}$, at least on one of the two components $\mathcal{A}_{1}$ or $\mathcal{A}_{2}$. Given that we are interested in measuring the entanglement between $\mathcal{A}_{1}$ and $\mathcal{A}_{2}$, the contributions from $\bar{g}$ and from $\overline{\bar{g}}$ are thence one and the same, and the results obtained for a single tensor product state remain unchanged.

On the contrary, when neither condition is satisfiable, the two states $\bar{g}$ and $\overline{\bar{g}}$ give different contributions and we find that, in addition to the expected topological entropy, the von Neumann entropy and entanglement negativity pick up a contribution that depends on the choice of quantum superposition of $\bar{g}$ and $\overline{\bar{g}}$. In the case of a superposition $\alpha \bar{g}_{\mathcal{B}}\left|0_{\mathcal{B}}\right\rangle+\beta \overline{\bar{g}}_{\mathcal{B}}\left|0_{\mathcal{B}}\right\rangle$, we obtain in App. E the von Neumann entropy

$$
S_{\mathrm{vN}}^{\left(\mathcal{A}_{1}\right)}=\ln \frac{\left|G_{\mathcal{A}}\right|}{\left|G_{\mathcal{A}_{1}}\right|\left|G_{\mathcal{A}_{2}}\right|}-|\alpha|^{2} \ln |\alpha|^{2}-|\beta|^{2} \ln |\beta|^{2},
$$

and the logarithmic entanglement negativity

$$
\mathcal{E}=\ln \frac{\left|G_{\mathcal{A}}\right|}{\left|G_{\mathcal{A}_{1}}\right|\left|G_{\mathcal{A}_{2}}\right|}+2 \ln (|\alpha|+|\beta|) .
$$

In addition to the usual topological contribution, a new term appears that directly depends on the weights of the states in the superposition. In Eq. (24) this takes the form of the classical entropy of mixing (recall that $|\alpha|^{2}+|\beta|^{2}=1$ ); however, the presence of a similar term in the negativity confirms that it originates from quantum rather than classical correlations. It is interesting to notice that the additional contribution takes a similar but not identical form in $S_{\mathrm{vN}}^{\left(\mathcal{A}_{1}\right)}$, Eq. (24), and in $\mathcal{E}$, Eq. (25).

A few comments are in order. Firstly, we note that the additional contribution is always positive. Therefore, the topological entropy $\gamma$ is a lower bound for the von Neumann entropy and entanglement negativity approaches proposed in this paper, with respect to the choice of projected state for subsystem $\mathcal{B}$. It may be possible to devise an appropriate minimisation routine on the latter to extract $\gamma$ without a priori knowledge of the suitable choice(s) for $\left|\phi_{\mathcal{B}}\right\rangle$.

Secondly, when neither conditions in Eqs. (22) and (23) are satisfied, we see from the calculation in App. E, e.g., Eq. (E26), that the two states identified by $\bar{g}$ and $\overline{\bar{g}}$ contribute separately and additively to the entanglement measure (be it the von Neumann entropy or the negativity). Therefore, the projection of subsystem $\mathcal{B}$ onto the (equal amplitude) superposition of these states is akin to taking the trace over them. The corresponding additional contribution amounts to the logarithm of the number of such states. One can verify that Eqs. (22) and (23) are not satisfied only if $\bar{g}$ and $\overline{\bar{g}}$ differ by the action of a (product of) star operators acting at the boundaries $\partial_{\mathcal{A}_{1}}$ and $\partial_{\mathcal{A}_{2}}$. Their number scales exponentially in the length of the boundaries, and this recovers indeed the area law contribution to the entanglement measures upon tracing rather than projecting onto a given state of subsystem $\mathcal{B}$.

\section{CLASSICAL VS QUANTUM ENTROPY}

In general, the von Neumann entropy is sensitive to both classical and quantum correlations. On the contrary, the entanglement negativity is a measure of quantum correlations alone. For this reason, it is interesting to see how their behaviour differs, in the context of the approach discussed in the present paper, for a classical topologically ordered system such as for instance the eight-vertex model [18] (see Ref. 14 for a calculation of the von Neumann entropy of this system).

The classical eight-vertex model is a combinatorial problem of arrows on the bonds of the square lattice, with the hard constraint that the number of incoming arrows at every vertex is even. As discussed in App. C, the density matrix of the toric code model, in the totally mixed limit where it is stripped of all its off-diagonal elements, reduces to the density matrix of the classical eight-vertex model. From this we obtain the (normalised) projected density matrix

$$
\rho_{\mathcal{A}}^{8 \mathrm{v}}=\frac{1}{\left|G_{\mathcal{A}}\right|} \sum_{g \in G_{\mathcal{A}}} g_{\mathcal{A}}\left|0_{\mathcal{A}}\right\rangle\left\langle 0_{\mathcal{A}}\right| g_{\mathcal{A}}
$$

Following the same steps used earlier in the von Neumann entropy calculation for the quantum case, we introduce $\rho_{\mathcal{A}_{1}}^{8 \mathrm{v}}$ by tracing over the degrees of freedom in $\mathcal{A}_{2}$,

$$
\rho_{\mathcal{A}_{1}}^{8 \mathrm{v}}=\frac{1}{\left|G_{\mathcal{A}}\right|} \sum_{g \in G_{\mathcal{A}}} g_{\mathcal{A}_{1}}\left|0_{\mathcal{A}_{1}}\right\rangle\left\langle 0_{\mathcal{A}_{1}}\right| g_{\mathcal{A}_{1}}
$$


and we compute

$$
\begin{aligned}
\left(\rho_{\mathcal{A}_{1}}^{8 \mathrm{v}}\right)^{2} & =\frac{1}{\left|G_{\mathcal{A}}\right|^{2}} \sum_{g, g^{\prime} \in G_{\mathcal{A}}} \\
& \times g_{\mathcal{A}_{1}}\left|0_{\mathcal{A}_{1}}\right\rangle\left\langle 0_{\mathcal{A}_{1}}\left|g_{\mathcal{A}_{1}} g_{\mathcal{A}_{1}}^{\prime}\right| 0_{\mathcal{A}_{1}}\right\rangle\left\langle 0_{\mathcal{A}_{1}}\right| g_{\mathcal{A}_{1}}^{\prime} \\
& =\frac{\left|G_{\mathcal{A}_{2}}\right|}{\left|G_{\mathcal{A}}\right|^{2}} \sum_{g \in G_{\mathcal{A}}} g_{\mathcal{A}_{1}}\left|0_{\mathcal{A}_{1}}\right\rangle\left\langle 0_{\mathcal{A}_{1}}\right| g_{\mathcal{A}_{1}} \\
& =\frac{\left|G_{\mathcal{A}_{2}}\right|}{\left|G_{\mathcal{A}}\right|} \rho_{\mathcal{A}_{1}}^{8 \mathrm{v}},
\end{aligned}
$$

where we made the change of variable $\tilde{g}=g^{\prime} g$, and then realised that the expectation value imposes $\tilde{g} \in G_{\mathcal{A}_{2}}$ and the dependence on $\tilde{g}$ disappears.

Iterating this identity, we obtain

$$
\operatorname{Tr}\left[\left(\rho_{\mathcal{A}_{1}}^{8 \mathrm{v}}\right)^{2}\right]=\left(\frac{\left|G_{\mathcal{A}_{2}}\right|}{\left|G_{\mathcal{A}}\right|}\right)^{n-1}
$$

and

$$
S_{\mathrm{vN}}^{\left(\mathcal{A}_{1}\right)}=-\ln \left(\frac{\left|G_{\mathcal{A}_{2}}\right|}{\left|G_{\mathcal{A}}\right|}\right)=\ln \left(2^{N_{\mathcal{A}_{1}}^{(s)}}\right)+\ln \left(2^{m_{\mathcal{B}}-1}\right),
$$

where we used again the fact that $\left|G_{\mathcal{A}_{2}}\right|=2^{N_{\mathcal{A}_{2}}^{(2)}}$ and $\left|G_{\mathcal{A}}\right|=$ $2^{N_{\mathcal{A}}^{(2)}+m_{\mathcal{B}}-1}$ ( $m_{\mathcal{B}}=2$ for the choice of partitions in Fig. 1). The first contribution is the expected extensive term (recall that the von Neumann entropy becomes a measure of the statistical mechanical entropy for classical systems) whereas the second (order one) contribution is a signature of the classical topologically ordered nature of the system.

Whereas the proposed approach via projecting the degrees of freedom in $\mathcal{B}$ no longer leads to a distillation of the sole topological contribution, we observe nonetheless a signature of the classical topological entropy of the system.

Let us contrast this result with the negativity calculation for the same system. Instead of tracing over $\mathcal{A}_{2}$, we take the transpose, which however leaves a purely diagonal density matrix unchanged, $\left(\rho_{\mathcal{A}}^{8 \mathrm{v}}\right)^{T_{2}} \equiv \rho_{\mathcal{A}}^{8 \mathrm{v}}$. The calculation of its square becomes therefore straightforward,

$$
\begin{aligned}
\left(\rho_{\mathcal{A}}^{8 \mathrm{v}}\right)^{2} & =\frac{1}{\left|G_{\mathcal{A}}\right|^{2}} \sum_{g, g^{\prime} \in G_{\mathcal{A}}} g_{\mathcal{A}}\left|0_{\mathcal{A}}\right\rangle\left\langle 0_{\mathcal{A}}\left|g_{\mathcal{A}} g_{\mathcal{A}}^{\prime}\right| 0_{\mathcal{A}}\right\rangle\left\langle 0_{\mathcal{A}}\right| g_{\mathcal{A}}^{\prime} \\
& =\frac{1}{\left|G_{\mathcal{A}}\right|} \rho_{\mathcal{A}}^{8 \mathrm{v}}
\end{aligned}
$$

since the expectation value selects $g^{\prime}$ uniquely by imposing the condition $g^{\prime}=g$. From this result we see that $\operatorname{Tr}\left[\left(\rho_{\mathcal{A}}^{8 \mathrm{v}}\right)^{n}\right]=1 /\left|G_{\mathcal{A}}\right|^{n-1}$, which has the same analytic continuation for $n$ even or odd and therefore the negativity vanishes, as expected for a system with purely classical correlations.

In summary, the calculation of the von Neumann entropy for a classical system contains a contribution due to the classical topological entropy, which is lost (as expected) in the negativity calculation. The clear difference in behaviour of the proposed von Neumann and negativity measures between quantum and classical systems can thus be used to distinguish between the two instances of topological order.

\section{CONCLUSIONS}

Obtaining the topological entropy of a system from analytical calculations or numerical simulations is in general a tall order, since it appears in measures of entanglement as an order one correction to leading terms that scale with the size of the system.

In this paper we have shown that one can distil the topological entropy alone using von Neumann entropy or entanglement negativity measures where the conventional trace over part of the system is replaced with a projection. The combination of such projection (the state of which needs not be known) with an appropriate choice of partitions allows to remove the unwanted boundary terms. The topological entropy of a system can thus be obtained via a single measurement, without the conventional need for elaborate subtraction schemes or extrapolation as a function of system size.

Given that the entanglement entropy computed from $\rho_{\mathcal{A}_{1}}$ in Eq. (11) is devoid of a boundary term, and yet not topologically trivial, it will be interesting to compare the corresponding entanglement spectrum with the one from the reduced density matrix obtained by tracing over both $\mathcal{A}_{2}$ and $\mathcal{B}$. This is however beyond the scope of the present paper.

In the paper, we also discussed the conditions that the projected wavefunction for $\mathcal{B}$ has to satisty in order for our results to hold. For instance, any tensor product state in the $\sigma^{z}$ or $\sigma^{x}$ basis is suitable. However, superpositions thereof require appropriate relations between the states involved, which are summarised by Eq. (22) and Eq. (23). When the conditions are violated, the von Neumann entropy and entanglement negativity acquire additional contributions on top of the expected topological entropy, which depend on the amplitudes of the states in the superposition. The additional contributions are always positive; it may therefore be possible to combine an appropriate minimisation procedure over the projected state with the approach in this paper to find suitable states where these contributions vanish.

We further showed that similar results apply to instances of classical topological order, provided that we use the von Neumann entanglement entropy. In this case, the topological correlations are purely classical in nature and the negativity vanishes identically. The combined use of both the von Neumann entropy and of the entanglement negativity allows thus to distinguish and characterise classical and quantum topolgical correlations.

Here we considered only the toric code at zero-temperature in two dimensions. From the results in Refs. 19 and 20, one expects the topological entropy to vanish in this system at any finite temperature in the thermodynamic limit. On the contrary, the toric code in $3 \mathrm{D}$ reduces to a classical $\mathbb{Z}_{2}$ gauge theory at finite temperature, which remains topologically ordered albeit only classically up to a finite temperature phase transition. Using the approach discussed in this paper and the results from both the von Neumann entropy and the entanglement negativity, one ought to observe that the former survives up to the transition (as a measure of classical topological entropy), whereas the latter vanishes at any finite temperature (as a measure of quantum topological entropy only). 
This is to be contrasted with, say, the $3 \mathrm{D}$ toric code at zero temperature in presence of a magnetic field, where both measures of the topological entropy survive as the field is increased, up to a quantum phase transition at finite field.

It will be interesting to see how the finite size behaviour of the negativity at finite field / finite temperature differs, and in particular whether the finite size behaviour in the negativity calculations of the quantum topological entropy bears a signature of the zero temperature topological order at sufficiently small but finite temperatures (cf. for example the finite size behaviour of the entanglement entropy in Refs. 19 and 20). The ability to compute only the topological entropy without boundary terms scaling with the size of the system may help to study these different scenarios.

Similar calculations could be extended to other 3D systems that have been recently argued to exhibit $\mathbb{Z}_{2}$ quantum spin liquid behaviour at finite temperature [21-23]. This would allow to test whether quantum topological order in such systems does indeed survive at finite temperature (as measured by the topological contribution to the entanglement), or it vanishes in the thermodynamic limit as is the case for the $3 \mathrm{D}$ toric code.

To some extent the toric code is a rather special example of topological order with precisely 'zero-ranged' local correlations. It will be interesting to study extensions of these calculations of the topological entropy, perhaps numerically, to more complex examples of topologically non-trivial states.

One could investigate perturbations of the toric code introduced via stochastic matrix form decomposition [13], where the GS wavefunction is known exactly throughout the phase diagram. These perturbations introduce finite correlations and eventually drive the system across a so-called conformal critical point. It may also be possible to study the behaviour of the negativity at such critical points by means of conformal field theoretic techniques [14, 24, 25]. This work could lead the way to the even more interesting challenge of applying this approach to quantum Hall states and other topologically ordered phases of matter.

As a closing remark, one should remember that a nonvanishing topological entropy per se is not evidence of topological order but rather an indication that the system can exhibit topological order. For instance, if a non-local term was added to the Hamiltonian which selected uniquely one of the degenerate ground states, the results presented in this paper would remain unchanged. In this respect, we notice that there is a difference between the choice of partitions in the top and bottom panels in Fig. 1. The bottom panel represents a local test of the ability of the system to support topological order, since it only looks at topological correlations within the outer boundary of $\mathcal{A}_{2}$.

\section{ACKNOWLEDGMENTS}

We are grateful to G. Vidal and C. Chamon for useful discussions. This work was supported by EPSRC Grant EP/K028960/1, and in part by the Helmholtz Virtual Institute "New States of Matter and Their Excitations" and by the EPSRC NetworkPlus on "Emergence and Physics far from Equi- librium".

\section{Appendix A: Projected $\rho_{\mathcal{A}}$ (quantum)}

Let us consider the density matrix of the system prepared in the GS in Eq. (2),

$$
\rho=\left|\psi_{0}\right\rangle\left\langle\psi_{0}\left|=\frac{1}{|G|} \sum_{g, g^{\prime} \in G} g\right| 0\right\rangle\langle 0| g^{\prime}
$$

and compute the projected density matrix

$$
\rho_{\mathcal{A}}=\left\langle\phi_{\mathcal{B}}|\rho| \phi_{\mathcal{B}}\right\rangle
$$

where $\left|\phi_{\mathcal{B}}\right\rangle=\bar{g}_{\mathcal{B}}\left|0_{\mathcal{B}}\right\rangle$, for some $\bar{g} \in G$.

It is convenient to redefine $g \rightarrow g \bar{g}$ and $g^{\prime} \rightarrow g^{\prime} \bar{g}$, which are one-to-one mappings in $G$. Using the fact that $(\bar{g})^{2}=1$ (and equivalently $\left(\bar{g}_{\mathcal{B}}\right)^{2}=\mathbf{1}_{\mathcal{B}}$ ), the projected density matrix can then be written as

$$
\begin{aligned}
\rho_{\mathcal{A}} & =\frac{1}{|G|} \sum_{g, g^{\prime} \in G} g_{\mathcal{A}} \bar{g}_{\mathcal{A}}\left|0_{\mathcal{A}}\right\rangle\left\langle 0_{\mathcal{A}}\right| \bar{g}_{\mathcal{A}} g_{\mathcal{A}}^{\prime} \\
& \times\left\langle 0_{\mathcal{B}}\left|g_{\mathcal{B}}\right| 0_{\mathcal{B}}\right\rangle\left\langle 0_{\mathcal{B}}\left|g_{\mathcal{B}}^{\prime}\right| 0_{\mathcal{B}}\right\rangle,
\end{aligned}
$$

where we introduced the notation $|0\rangle=\left|0_{\mathcal{A}}\right\rangle \otimes\left|0_{\mathcal{B}}\right\rangle$ and $g=g_{\mathcal{A}} \otimes g_{\mathcal{B}}$. The two expectation values impose that $g_{\mathcal{B}}=g_{\mathcal{B}}^{\prime}=\mathbf{1}_{\mathcal{B}}$, i.e., $g, g^{\prime} \in G_{\mathcal{A}} \subset G$. At the same time, we can redefine the reference state $|0\rangle \rightarrow \bar{g}|0\rangle$ (recall that the choice of reference state in Eq. (2) was arbitrary) and we arrive at the expression

$$
\rho_{\mathcal{A}}=\frac{1}{|G|} \sum_{g, g^{\prime} \in G_{\mathcal{A}}} g_{\mathcal{A}}\left|0_{\mathcal{A}}\right\rangle\left\langle 0_{\mathcal{A}}\right| g_{\mathcal{A}}^{\prime}
$$

For convenience, we further normalise $\rho_{\mathcal{A}}$ by replacing the factor $1 /|G|$ with $1 /\left|G_{\mathcal{A}}\right|$.

\section{Appendix B: Entanglement entropy of the projected $\rho_{\mathcal{A}}$}

It is straightforward to show that the von Neumann entropy of subsystem $\mathcal{A}$ vanishes once $\mathcal{B}$ has been projected to a given state $\bar{g}_{\mathcal{B}}\left|0_{\mathcal{B}}\right\rangle$. In order to obtain the von Neumann entropy of $\rho_{\mathcal{A}}$ in Eq. (A4) (equivalently, Eq. (9) in the main text), we need to compute

$\rho_{\mathcal{A}}^{2}=\frac{1}{\left|G_{\mathcal{A}}\right|^{2}} \sum_{g, g^{\prime} \in G_{\mathcal{A}}} \sum_{\tilde{g}, \tilde{g}^{\prime} \in G_{\mathcal{A}}} g_{\mathcal{A}}\left|0_{\mathcal{A}}\right\rangle\left\langle 0_{\mathcal{A}}\left|g_{\mathcal{A}}^{\prime} \tilde{g}_{\mathcal{A}}\right| 0_{\mathcal{A}}\right\rangle\left\langle 0_{\mathcal{A}}\right| \tilde{g}_{\mathcal{A}}^{\prime}$.

The product $g^{\prime} \tilde{g}$ is a generic element of $G_{\mathcal{A}}$, since both $g^{\prime}$ and $\tilde{g}$ belong to the same group. Therefore, the corresponding expectation value fixes uniquely the product to the identity $\mathbf{1}$, and we are left with a free summation over the elements of $G_{\mathcal{A}}$ (i.e., a factor of $\left|G_{\mathcal{A}}\right|$ ):

$$
\rho_{\mathcal{A}}^{2}=\frac{1}{\left|G_{\mathcal{A}}\right|} \sum_{g \in G_{\mathcal{A}}} \sum_{\tilde{g}^{\prime} \in G_{\mathcal{A}}} g_{\mathcal{A}}\left|0_{\mathcal{A}}\right\rangle\left\langle 0_{\mathcal{A}}\right| \tilde{g}_{\mathcal{A}}^{\prime}=\rho_{\mathcal{A}}
$$

Iterating this identity, one finds that $\rho_{\mathcal{A}}^{n}=\rho_{\mathcal{A}}, \operatorname{Tr}\left(\rho_{\mathcal{A}}^{n}\right)=1$, and $S_{\mathrm{vN}}^{(\mathcal{A})}=-\lim _{n \rightarrow 1} \partial_{n}\left[\operatorname{Tr}\left(\rho_{\mathcal{A}}^{n}\right)\right]=0$. 


\section{Appendix C: Projected $\rho_{\mathcal{A}}$ (classical)}

The classical eight-vertex model is a combinatorial problem of arrows on the bonds of the square lattice, with the hard constraint that the number of incoming arrows at every vertex is even (counting 0 as an even number). Taking advantage of the bipartite nature of the lattice, we can define arrows going from sublattice A to sublattice B as positive spins, and all others are negative. This establishes a 1-to-1 mapping between eight-vertex configurations and $\sigma^{z}$ tensor product states that minimise the energy of the plaquette term in the toric code Hamiltonian, Eq. (1). All eight-vertex configurations can be obtained from a reference configuration, say the spin polarized $|0\rangle$, by acting with elements of $G$. The eight-vertex model represents an instance of a classical topologically ordered system [14].

The density matrix of the toric code model, in the totally mixed limit where it is stripped of all its off-diagonal elements,

$$
\rho=\frac{1}{|G|} \sum_{g \in G} g|0\rangle\langle 0| g
$$

reduces thus to the density matrix of the classical eight-vertex model.

Let us compute the projected density matrix in this case, $\rho_{\mathcal{A}}=\left\langle\phi_{\mathcal{B}}|\rho| \phi_{\mathcal{B}}\right\rangle$, with $\left|\phi_{\mathcal{B}}\right\rangle=\bar{g}_{\mathcal{B}}\left|0_{\mathcal{B}}\right\rangle$, for some $\bar{g} \in G$. Using once again the change of summation variable $g \rightarrow g \bar{g}$, the projected density matrix can then be written as

$$
\begin{aligned}
\rho_{\mathcal{A}} & =\frac{1}{|G|} \sum_{g \in G} g_{\mathcal{A}} \bar{g}_{\mathcal{A}}\left|0_{\mathcal{A}}\right\rangle\left\langle 0_{\mathcal{A}}\right| \bar{g}_{\mathcal{A}} g_{\mathcal{A}} \\
& \times\left\langle 0_{\mathcal{B}}\left|g_{\mathcal{B}}\right| 0_{\mathcal{B}}\right\rangle\left\langle 0_{\mathcal{B}}\left|g_{\mathcal{B}}\right| 0_{\mathcal{B}}\right\rangle,
\end{aligned}
$$

where the two expectation values impose that $g_{\mathcal{B}}=\mathbf{1}_{\mathcal{B}}$, i.e., $g \in G_{\mathcal{A}}$. Once we redefine the reference state $|0\rangle \rightarrow \bar{g}|0\rangle$ (recall that the choice of reference state in Eq. (2) was indeed arbitrary), we arrive at the expression

$$
\rho_{\mathcal{A}}=\frac{1}{|G|} \sum_{g \in G_{\mathcal{A}}} g_{\mathcal{A}}\left|0_{\mathcal{A}}\right\rangle\left\langle 0_{\mathcal{A}}\right| g_{\mathcal{A}}
$$

As before, we finally normalise $\rho_{\mathcal{A}}$ by replacing the factor $1 /|G|$ with $1 /\left|G_{\mathcal{A}}\right|$.

\section{Appendix D: Negativity of the partitions in Fig. 1}

The entanglement negativity for the partitions shown in Fig. 1, after tracing over $\mathcal{B}$ and transposing $\mathcal{A}_{2}$, was computed in Refs. 8 and 9. Here we present a more streamlined version of the calculation in Ref. 9, which is at the basis of the results presented in the main text of the paper after projecting the degrees of freedom in $\mathcal{B}$.
We start with the density matrix of the system prepared in the GS in Eq. (2),

$$
\rho=\left|\psi_{0}\right\rangle\left\langle\psi_{0}\left|=\frac{1}{|G|} \sum_{g, g^{\prime} \in G} g\right| 0\right\rangle\langle 0| g^{\prime}
$$

and take the trace over the degrees of freedom in $\mathcal{B}$,

$$
\rho_{\mathcal{A}}=\frac{1}{|G|} \sum_{g, g^{\prime} \in G} g_{\mathcal{A}}\left|0_{\mathcal{A}}\right\rangle\left\langle 0_{\mathcal{A}}\right| g_{\mathcal{A}}^{\prime}\left\langle 0_{\mathcal{B}}\left|g_{\mathcal{B}}^{\prime} g_{\mathcal{B}}\right| 0_{\mathcal{B}}\right\rangle
$$

It is then convenient to redefine $g^{\prime} \rightarrow \tilde{g}=g^{\prime} g$ (one-to-one mapping in $G$ ) and replace the summation over $g^{\prime}$ with a summation over $\tilde{g}$. The expectation value then restricts $\tilde{g}$ to act as the identity on subsystem $\mathcal{B}$, i.e., $\tilde{g} \in G_{\mathcal{A}}$ :

$$
\rho_{\mathcal{A}}=\frac{1}{|G|} \sum_{g \in G, \tilde{g} \in G_{\mathcal{A}}} g_{\mathcal{A}}\left|0_{\mathcal{A}}\right\rangle\left\langle 0_{\mathcal{A}}\right| g_{\mathcal{A}} \tilde{g}_{\mathcal{A}} .
$$

Notice that this expression differs from the projected $\rho_{\mathcal{A}}$ Eq. (9) in that $g$ spans the whole group $G$ rather than its subgroup $G_{\mathcal{A}}$. This difference will however play a crucial role in the calculation of the negativity.

After taking the transpose over the degrees of freedom in $\mathcal{A}_{2}$,

$$
\begin{aligned}
\rho_{\mathcal{A}}^{T_{2}} & =\frac{1}{|G|} \sum_{g \in G, \tilde{g} \in G_{\mathcal{A}}} \\
& \times\left(g_{\mathcal{A}_{1}}\left|0_{\mathcal{A}_{1}}\right\rangle\left\langle 0_{\mathcal{A}_{1}}\right| g_{\mathcal{A}_{1}} \tilde{g}_{\mathcal{A}_{1}}\right) \\
& \otimes\left(g_{\mathcal{A}_{2}} \tilde{g}_{\mathcal{A}_{2}}\left|0_{\mathcal{A}_{2}}\right\rangle\left\langle 0_{\mathcal{A}_{2}}\right| g_{\mathcal{A}_{2}}\right) .
\end{aligned}
$$

we compute the second power of $\rho_{\mathcal{A}}^{T_{2}}$ :

$$
\begin{aligned}
& \left(\rho_{\mathcal{A}}^{T_{2}}\right)^{2}=\frac{1}{|G|^{2}} \sum_{g_{1} \in G} \sum_{\tilde{g}_{1} \in G} \sum_{g \in G} \sum_{\tilde{g} \in G_{\mathcal{A}}} \\
& \quad \times\left(g_{1 \mathcal{A}_{1}}\left|0_{\mathcal{A}_{1}}\right\rangle\left\langle 0_{\mathcal{A}_{1}}\left|g_{1 \mathcal{A}_{1}} \tilde{g}_{1 \mathcal{A}_{1}} g_{\mathcal{A}_{1}}\right| 0_{\mathcal{A}_{1}}\right\rangle\left\langle 0_{\mathcal{A}_{1}}\right| g_{\mathcal{A}_{1}} \tilde{g}_{\mathcal{A}_{1}}\right) \\
& \otimes\left(g_{1 \mathcal{A}_{2}} \tilde{g}_{1 \mathcal{A}_{2}}\left|0_{\mathcal{A}_{2}}\right\rangle\left\langle 0_{\mathcal{A}_{2}}\left|g_{1 \mathcal{A}_{2}} g_{\mathcal{A}_{2}} \tilde{g}_{\mathcal{A}_{2}}\right| 0_{\mathcal{A}_{2}}\right\rangle\left\langle 0_{\mathcal{A}_{2}}\right| g_{\mathcal{A}_{2}}\right) .
\end{aligned}
$$

One can replace the summation over $g$ with a summation over $g_{2}=g_{1} g \in G$, upon substituting $g=g_{1} g_{2}$ in the expression above (one-to-one mapping in $G$, given $g_{1}$ ). If we also relabel $\tilde{g} \equiv \tilde{g}_{2}$, we obtain the following expression:

$$
\begin{aligned}
& \left(\rho_{\mathcal{A}}^{T_{2}}\right)^{2}=\frac{1}{|G|^{2}} \sum_{g_{1}, g_{2} \in G} \sum_{\tilde{g}_{1}, \tilde{g}_{2} \in G_{\mathcal{A}}} \\
& \times\left(g_{1 \mathcal{A}_{1}}\left|0_{\mathcal{A}_{1}}\right\rangle\left\langle 0_{\mathcal{A}_{1}}\left|g_{2 \mathcal{A}_{1}} \tilde{g}_{1 \mathcal{A}_{1}}\right| 0_{\mathcal{A}_{1}}\right\rangle\left\langle 0_{\mathcal{A}_{1}}\right| g_{1 \mathcal{A}_{1}} g_{2 \mathcal{A}_{1}} \tilde{g}_{2 \mathcal{A}_{1}}\right) \\
& \otimes\left(g_{1 \mathcal{A}_{2}} \tilde{g}_{1 \mathcal{A}_{2}}\left|0_{\mathcal{A}_{2}}\right\rangle\left\langle 0_{\mathcal{A}_{2}}\left|g_{2 \mathcal{A}_{2}} \tilde{g}_{2 \mathcal{A}_{2}}\right| 0_{\mathcal{A}_{2}}\right\rangle\left\langle 0_{\mathcal{A}_{2}}\right| g_{1 \mathcal{A}_{2}} g_{2 \mathcal{A}_{2}}\right) .
\end{aligned}
$$

Similar considerations lead to the third power of $\rho_{\mathcal{A}}^{T_{2}}$, from which one can recognise the general pattern: 


$$
\begin{aligned}
\left(\rho_{\mathcal{A}}^{T_{2}}\right)^{n} & =\frac{1}{|G|^{n}} \sum_{g_{1}, \ldots, g_{n} \in G} \sum_{\tilde{g}_{1}, \ldots, \tilde{g}_{n} \in G_{\mathcal{A}}} \\
& \times\left[g_{1 \mathcal{A}_{1}}\left|0_{\mathcal{A}_{1}}\right\rangle \prod_{\ell=2}^{n}\left\langle 0_{\mathcal{A}_{1}}\left|g_{\ell \mathcal{A}_{1}} \tilde{g}_{(\ell-1) \mathcal{A}_{1}}\right| 0_{\mathcal{A}_{1}}\right\rangle\left\langle 0_{\mathcal{A}_{1}}\right|\left(\prod_{\ell=1}^{n} g_{\ell \mathcal{A}_{1}}\right) \tilde{g}_{n \mathcal{A}_{1}}\right] \\
& \otimes\left[g_{1 \mathcal{A}_{2}} \tilde{g}_{1 \mathcal{A}_{2}}\left|0_{\mathcal{A}_{2}}\right\rangle \prod_{\ell=2}^{n}\left\langle 0_{\mathcal{A}_{2}}\left|g_{\ell \mathcal{A}_{2}} \tilde{g}_{\ell \mathcal{A}_{2}}\right| 0_{\mathcal{A}_{2}}\right\rangle\left\langle 0_{\mathcal{A}_{2}}\right| \prod_{\ell=1}^{n} g_{\ell \mathcal{A}_{2}}\right] .
\end{aligned}
$$

Upon taking the trace, one notices that the dependence on $g_{1}$ disappears, thus resulting in an overall factor $|G|$ :

$$
\begin{aligned}
\operatorname{Tr}\left[\left(\rho_{\mathcal{A}}^{T_{2}}\right)^{n}\right] & =\frac{1}{|G|^{n-1}} \sum_{g_{2}, \ldots, g_{n} \in G} \sum_{\tilde{g}_{1}, \ldots, \tilde{g}_{n} \in G_{\mathcal{A}}} \\
& \times \prod_{\ell=2}^{n}\left\langle 0_{\mathcal{A}_{1}}\left|g_{\ell \mathcal{A}_{1}} \tilde{g}_{(\ell-1) \mathcal{A}_{1}}\right| 0_{\mathcal{A}_{1}}\right\rangle\left\langle 0_{\mathcal{A}_{1}}\left|\left(\prod_{\ell=2}^{n} g_{\ell \mathcal{A}_{1}}\right) \tilde{g}_{n \mathcal{A}_{1}}\right| 0_{\mathcal{A}_{1}}\right\rangle \\
& \times \prod_{\ell=2}^{n}\left\langle 0_{\mathcal{A}_{2}}\left|g_{\ell \mathcal{A}_{2}} \tilde{g}_{\ell \mathcal{A}_{2}}\right| 0_{\mathcal{A}_{2}}\right\rangle\left\langle 0_{\mathcal{A}_{2}}\left|\left(\prod_{\ell=2}^{n} g_{\ell \mathcal{A}_{2}}\right) \tilde{g}_{1 \mathcal{A}_{2}}\right| 0_{\mathcal{A}_{2}}\right\rangle .
\end{aligned}
$$

Moreover, the first $n-1$ expectation values in the second and third rows of Eq. (D8) impose that $g_{\ell \mathcal{A}_{1}}=\tilde{g}_{(\ell-1) \mathcal{A}_{1}}$ and $g_{\ell \mathcal{A}_{2}}=\tilde{g}_{\ell \mathcal{A}_{2}}$, for $\ell=2, \ldots, n$. Using these identities in the last expectation value in each row, the expression above reduces to

$$
\begin{aligned}
& \operatorname{Tr}\left[\left(\rho_{\mathcal{A}}^{T_{2}}\right)^{n}\right]=\frac{1}{|G|^{n-1}} \sum_{g_{2}, \ldots, g_{n} \in G} \sum_{\tilde{g}_{1}, \ldots, \tilde{g}_{n} \in G_{\mathcal{A}}} \\
& \times \prod_{\ell=2}^{n}\left\langle 0_{\mathcal{A}_{1}}\left|g_{\ell \mathcal{A}_{1}} \tilde{g}_{(\ell-1) \mathcal{A}_{1}}\right| 0_{\mathcal{A}_{1}}\right\rangle\left\langle 0_{\mathcal{A}_{1}}\left|\prod_{\ell=1}^{n} \tilde{g}_{\ell \mathcal{A}_{1}}\right| 0_{\mathcal{A}_{1}}\right\rangle \\
& \times \prod_{\ell=2}^{n}\left\langle 0_{\mathcal{A}_{2}}\left|g_{\ell \mathcal{A}_{2}} \tilde{g}_{\ell \mathcal{A}_{2}}\right| 0_{\mathcal{A}_{2}}\right\rangle\left\langle 0_{\mathcal{A}_{2}}\left|\prod_{\ell=1}^{n} \tilde{g}_{\ell \mathcal{A}_{2}}\right| 0_{\mathcal{A}_{2}}\right\rangle .
\end{aligned}
$$

The last expectation values on each row, combined, impose that the product $\prod_{\ell=1}^{n} \tilde{g}_{\ell}$ acts as the identity on $\mathcal{A}$, which fixes uniquely one of the $\tilde{g}_{\ell}$, since they are all elements of $G_{\mathcal{A}}$. The remaining $2(n-1)$ expectation values impose

$$
g_{\ell \mathcal{A}}=\tilde{g}_{(\ell-1) \mathcal{A}_{1}} \otimes \tilde{g}_{\ell \mathcal{A}_{2}}
$$

for all $\ell=2, \ldots, n$. If this condition can be met, then the only freedom left in the choice of $g_{\ell} \in G$ is the multiplication by a generic element in $G_{\mathcal{B}}$, which results in a factor $\left|G_{\mathcal{B}}\right|$ upon summation over $g_{\ell}$.

Whether the chosen density matrix and partitions have a vanishing or non-vanishing negativity is entirely dependent on whether there exists at least one element in $G$ that can satisfy the condition in Eq. (D10), for all $\ell=2, \ldots, n$. If the answer is positive, then the trace reduces to

$$
\operatorname{Tr}\left[\left(\rho_{\mathcal{A}}^{T_{2}}\right)^{n}\right]=\frac{\left|G_{\mathcal{A}}\right|^{n-1}\left|G_{\mathcal{B}}\right|^{n-1}}{|G|^{n-1}}
$$

and the negativity vanishes identically (no difference between the even and odd $n$ analytic continuations).
Let us consider the condition in Eq. (D10) in the context of the partitions in Fig 1. The group $G_{\mathcal{A}}$ can be decomposed as the product of three groups, $G_{\mathcal{A}_{1}}, G_{\mathcal{A}_{2}}$, and $G_{\mathcal{A}_{1} \mathcal{A}_{2}}$, where $G_{\mathcal{A}_{1}}$ and $G_{\mathcal{A}_{2}}$ act only on $\mathcal{A}_{1}$ and $\mathcal{A}_{2}$, respectively, and $G_{\mathcal{A}_{1} \mathcal{A}_{2}}$ is defined as the quotient group $G_{\mathcal{A}} /\left(G_{\mathcal{A}_{1}} G_{\mathcal{A}_{2}}\right)$. Each element $\tilde{g}_{\ell} \in G_{\mathcal{A}}$ can correspondingly be uniquely decomposed as the product $\tilde{g}_{\ell}^{(1)} \tilde{g}_{\ell}^{(2)} \tilde{g}_{\ell}^{(12)}$ of three elements from each of the subgroups.

In this notation, the condition in Eq. (D10) can be written as

$$
\begin{aligned}
g_{\ell \mathcal{A}} & =\tilde{g}_{(\ell-1) \mathcal{A}_{1}}^{(1)} \tilde{g}_{(\ell-1) \mathcal{A}_{1}}^{(12)} \otimes \tilde{g}_{\ell \mathcal{A}_{2}}^{(2)} \tilde{g}_{\ell \mathcal{A}_{2}}^{(12)} \\
& =\left(\tilde{g}_{(\ell-1) \mathcal{A}_{1}}^{(1)} \otimes \tilde{g}_{\ell \mathcal{A}_{2}}^{(2)}\right)\left(\tilde{g}_{(\ell-1) \mathcal{A}_{1}}^{(12)} \otimes \tilde{g}_{\ell \mathcal{A}_{2}}^{(12)}\right) .
\end{aligned}
$$

The first of the two factors is trivial since it is nothing but the product of $\tilde{g}_{\ell-1}^{(1)} \in G_{\mathcal{A}_{1}}$ times $\tilde{g}_{\ell}^{(2)} \in G_{\mathcal{A}_{2}}$, which is an element of $G$ and thus can be matched by an appropriate choice of $g_{\ell}$. On the other hand, the second product $\tilde{g}_{(\ell-1) \mathcal{A}_{1}}^{(12)} \otimes \tilde{g}_{\ell \mathcal{A}_{2}}^{(12)}$ is not obviously the action on $\mathcal{A}$ of an element of $G$, and one ought to consider it with care.

With the choice in Fig. 1, the group $G_{\mathcal{A}_{1} \mathcal{A}_{2}}$ has only two elements: the identity, and the product $\theta$ of all star operators that act on at least one spin in $\mathcal{B}_{1}$. One can see that this element $\theta \in G$ acts simultaneously on $\mathcal{A}_{1}$ and $\mathcal{A}_{2}$ and cannot be written as a product of star operators acting solely (and separately) on spins in $\mathcal{A}_{1}$ and $\mathcal{A}_{2}$. With two choices each for $\tilde{g}_{\ell-1}^{(12)}$ and $\tilde{g}_{\ell}^{(12)}$, the non trivial option that requires consideration is when one of them is equal to $\theta$ and the other is the identity. In this case, we need to ask whether an element $g_{\ell} \in G$ exists such that $g_{\ell \mathcal{A}_{1}}=\theta_{\mathcal{A}_{1}}$ and $g_{\ell \mathcal{A}_{2}}=\mathbf{1}_{\mathcal{A}_{2}}$ (or vice versa).

The action of $\theta_{\mathcal{A}_{1}}$ is to flip all the spins in $\mathcal{A}_{1}$ that belong to stars at the boundary with $\mathcal{B}_{1}$. The product of all the corresponding boundary star operators is clearly an element $g^{*} \in G$ that acts on the system by flipping all the spins flipped by $\theta_{\mathcal{A}_{1}}$ as well as some spins in $\mathcal{B}_{1}$. Therefore, 
$g_{\mathcal{A}}^{*}=\theta_{\mathcal{A}_{1}} \otimes \mathbf{1}_{\mathcal{A}_{2}}$ and the condition in Eq. (D10) is satisfied. Since this constructive argument applies in general for the choice of partitions in Fig. 1, we conclude that the negativity vanishes identically.

\section{Appendix E: Projection onto a generic state}

In the main text we considered the case where subsystem $\mathcal{B}$ is projected onto one of the $\sigma^{z}$ tensor product states. Here we discuss the case of a generic state, which can always be written as a superposition of $\sigma^{z}$ tensor product states. We show that the results obtained earlier hold only for a special class of superpositions, and otherwise the entanglement entropy becomes a function of both the topological contribution and of the amplitudes in the projected wavefunction.

Notice that, for a generic superposition

$$
\left|\phi_{\mathcal{B}}\right\rangle=\sum_{\left\{\sigma_{i}^{z}\right\}_{\mathcal{B}}} \alpha\left(\left\{\sigma_{i}^{z}\right\}_{\mathcal{B}}\right)\left|\left\{\sigma_{i}^{z}\right\}_{\mathcal{B}}\right\rangle
$$

the only states that give a non-vanishing contribution to the projection of the density matrix of the system prepared in the state $\left|\psi_{0}\right\rangle$, Eq. (2), are those where $\left|\left\{\sigma_{i}^{z}\right\}_{\mathcal{B}}\right\rangle=\bar{g}_{\mathcal{B}}\left|0_{\mathcal{B}}\right\rangle$ for some $\bar{g} \in G$. For simplicity, we focus on the case of a coherent superposition between only two such states, which is sufficient for the purpose of this discussion:

$$
\left|\phi_{\mathcal{B}}\right\rangle=\alpha \bar{g}_{\mathcal{B}}\left|0_{\mathcal{B}}\right\rangle+\beta \overline{\bar{g}}_{\mathcal{B}}\left|0_{\mathcal{B}}\right\rangle
$$

The state is assumed normalised, $|\alpha|^{2}+|\beta|^{2}=1$.

The (normalised) projected density matrix can then be written as

$$
\begin{aligned}
\rho_{\mathcal{A}} & =\frac{1}{\mathcal{N}} \sum_{g, g^{\prime} \in G}\left(\alpha^{*}\left\langle 0_{\mathcal{B}}\left|\bar{g}_{\mathcal{B}}+\beta^{*}\left\langle 0_{\mathcal{B}}\right| \overline{\bar{g}}_{\mathcal{B}}\right) g_{\mathcal{B}} \mid 0_{\mathcal{B}}\right\rangle\right. \\
& \times\left\langle 0_{\mathcal{B}}\left|g_{\mathcal{B}}^{\prime}\left(\alpha \bar{g}_{\mathcal{B}}\left|0_{\mathcal{B}}\right\rangle+\beta \overline{\bar{g}}_{\mathcal{B}}\left|0_{\mathcal{B}}\right\rangle\right) g_{\mathcal{A}}\right| 0_{\mathcal{A}}\right\rangle\left\langle 0_{\mathcal{A}}\right| g_{\mathcal{A}}^{\prime}
\end{aligned}
$$

Using a simplified notation to save space (the state $0_{\mathcal{B}}$ and the subscripts $\mathcal{B}$ are understood), the two matrix elements can be expanded as

$$
\begin{aligned}
& \left(\alpha ^ { * } \langle \overline { g } | + \beta ^ { * } \langle \overline { \overline { g } } | ) | g \rangle \left\langleg^{\prime}|(\alpha|\bar{g}\rangle+\beta|\overline{\bar{g}}\rangle)\right.\right. \\
& \quad=\left[\alpha^{*}\langle\bar{g} g\rangle+\beta^{*}\langle\overline{\bar{g}} g\rangle\right]\left[\alpha\left\langle g^{\prime} \bar{g}\right\rangle+\beta\left\langle g^{\prime} \overline{\bar{g}}\right\rangle\right] \\
& \quad=|\alpha|^{2}\langle\bar{g} g\rangle\left\langle g^{\prime} \bar{g}\right\rangle+\alpha^{*} \beta\langle\bar{g} g\rangle\left\langle g^{\prime} \overline{\bar{g}}\right\rangle \\
& \quad+\beta^{*} \alpha\langle\overline{\bar{g}} g\rangle\left\langle g^{\prime} \bar{g}\right\rangle+|\beta|^{2}\langle\overline{\bar{g}} g\rangle\left\langle g^{\prime} \overline{\bar{g}} .\right.
\end{aligned}
$$

We can then perform the summation in Eq. (E3) separately for each of the four terms, after changing variables so as to reduce the expectation values to the form $\langle g\rangle\left\langle g^{\prime}\right\rangle$ (for instance for the first term proportional to $|\alpha|^{2}$ one needs to redefine $g \rightarrow \bar{g} g$ and $g^{\prime} \rightarrow \bar{g} g^{\prime}$ ). The expectation values impose $g, g^{\prime} \in G_{\mathcal{A}}$, and we obtain the expression

$$
\begin{aligned}
\rho_{\mathcal{A}}=\frac{1}{\mathcal{N}} \sum_{g, g^{\prime} \in G_{\mathcal{A}}} & {\left[|\alpha|^{2} g_{\mathcal{A}} \bar{g}_{\mathcal{A}}\left|0_{\mathcal{A}}\right\rangle\left\langle 0_{\mathcal{A}}\right| \bar{g}_{\mathcal{A}} g_{\mathcal{A}}^{\prime}\right.} \\
& +\alpha^{*} \beta g_{\mathcal{A}} \bar{g}_{\mathcal{A}}\left|0_{\mathcal{A}}\right\rangle\left\langle 0_{\mathcal{A}}\right| \overline{\bar{g}}_{\mathcal{A}} g_{\mathcal{A}}^{\prime} \\
& +\beta^{*} \alpha g_{\mathcal{A}} \overline{\bar{g}}_{\mathcal{A}}\left|0_{\mathcal{A}}\right\rangle\left\langle 0_{\mathcal{A}}\right| \bar{g}_{\mathcal{A}} g_{\mathcal{A}}^{\prime} \\
& \left.+|\beta|^{2} g_{\mathcal{A}} \overline{\bar{g}}_{\mathcal{A}}\left|0_{\mathcal{A}}\right\rangle\left\langle 0_{\mathcal{A}}\right| \overline{\bar{g}}_{\mathcal{A}} g_{\mathcal{A}}^{\prime}\right]
\end{aligned}
$$

The normalisation factor is determined by requiring that $\operatorname{Tr} \rho_{\mathcal{A}}=1$, which is straightforwardly obtained from Eq. (E5):

$$
\mathcal{N}=\left|G_{\mathcal{A}}\right|\left[1+x \sum_{g \in G_{\mathcal{A}}}\left\langle 0_{\mathcal{A}}\left|g_{\mathcal{A}} \bar{g}_{\mathcal{A}} \overline{\bar{g}}_{\mathcal{A}}\right| 0_{\mathcal{A}}\right\rangle\right],
$$

where we used the fact that $|\alpha|^{2}+|\beta|^{2}=1$ and we defined $x=\alpha^{*} \beta+\beta^{*} \alpha$ for brevity. Notice that the expectation value in the normalisation factor either fixes $g_{\mathcal{A}}$ uniquely if $\bar{g}_{\mathcal{A}} \overline{\bar{g}}_{\mathcal{A}} \otimes$ $\mathbf{1}_{\mathcal{B}} \in G$, or it vanishes identically otherwise.

Let us consider first the case where an element $g^{*}=$ $\bar{g}_{\mathcal{A}} \overline{\bar{g}}_{\mathcal{A}} \otimes \mathbf{1}_{\mathcal{B}}$ in $G$ exists (and therefore $g^{*} \in G_{\mathcal{A}}$ ). We proceed to take the trace over subsystem $\mathcal{A}_{2}$ term by term from Eq. (E5), leading to the expectation values

$$
\begin{array}{r}
\left\langle 0_{\mathcal{A}_{2}}\left|g_{\mathcal{A}_{2}}^{\prime} g_{\mathcal{A}_{2}}\right| 0_{\mathcal{A}_{2}}\right\rangle \\
\left\langle 0_{\mathcal{A}_{2}}\left|\overline{\bar{g}}_{\mathcal{A}_{2}} g_{\mathcal{A}_{2}}^{\prime} g_{\mathcal{A}_{2}} \bar{g}_{\mathcal{A}_{2}}\right| 0_{\mathcal{A}_{2}}\right\rangle \\
\left\langle 0_{\mathcal{A}_{2}}\left|\bar{g}_{\mathcal{A}_{2}} g_{\mathcal{A}_{2}}^{\prime} g_{\mathcal{A}_{2}} \overline{\bar{g}}_{\mathcal{A}_{2}}\right| 0_{\mathcal{A}_{2}}\right\rangle \\
\left\langle 0_{\mathcal{A}_{2}}\left|g_{\mathcal{A}_{2}}^{\prime} g_{\mathcal{A}_{2}}\right| 0_{\mathcal{A}_{2}}\right\rangle .
\end{array}
$$

The first and last expectation values can always be satisfied and they impose $g^{\prime}=g \tilde{g}$, for any $\tilde{g} \in G_{\mathcal{A}_{1}}$. On the contrary, the second and third expectation values can be satisfied only if an element $h \in G_{\mathcal{A}}$ exists such that $h_{\mathcal{A}_{2}}=\bar{g}_{\mathcal{A}_{2}} \overline{\bar{g}}_{\mathcal{A}_{2}}$. Our assumption of the existence of $g^{*} \in G_{\mathcal{A}}$ clearly satisfies this condition for $h=g^{*}$. However, we notice that this is a sufficient but not necessary assumption, and we shall comment more on this later.

The existence of such element $g^{*}$ allows us to change the summation label $g^{\prime} \rightarrow g^{\prime} g g^{*}$ for any $g \in G_{\mathcal{A}}$, whereby:

$$
\begin{aligned}
\left\langle 0_{\mathcal{A}_{2}}\left|\overline{\bar{g}}_{\mathcal{A}_{2}} g_{\mathcal{A}_{2}}^{\prime} g_{\mathcal{A}_{2}} \bar{g}_{\mathcal{A}_{2}}\right| 0_{\mathcal{A}_{2}}\right\rangle & \rightarrow\left\langle 0_{\mathcal{A}_{2}}\left|g_{\mathcal{A}_{2}}^{\prime}\right| 0_{\mathcal{A}_{2}}\right\rangle \\
\overline{\bar{g}}_{\mathcal{A}_{1}} g_{\mathcal{A}_{1}}^{\prime} & \rightarrow \bar{g}_{\mathcal{A}_{1}} g_{\mathcal{A}_{1}} g_{\mathcal{A}_{1}}^{\prime} \\
\bar{g}_{\mathcal{A}_{1}} g_{\mathcal{A}_{1}}^{\prime} & \rightarrow \overline{\bar{g}}_{\mathcal{A}_{1}} g_{\mathcal{A}_{1}} g_{\mathcal{A}_{1}}^{\prime} .
\end{aligned}
$$

The new form of the expectation value in the first of the three expressions above straightforwardly imposes that $g^{\prime} \in G_{\mathcal{A}_{1}}$, and the reduced density matrix becomes

$$
\begin{aligned}
\rho_{\mathcal{A}_{1}} & =\frac{1}{\mathcal{N}} \sum_{g \in G_{\mathcal{A}}} \sum_{\tilde{g} \in G_{\mathcal{A}_{1}}} \\
& \times\left[\left(|\alpha|^{2}+\alpha^{*} \beta\right) g_{\mathcal{A}_{1}} \bar{g}_{\mathcal{A}_{1}}\left|0_{\mathcal{A}_{1}}\right\rangle\left\langle 0_{\mathcal{A}_{1}}\right| \bar{g}_{\mathcal{A}_{1}} g_{\mathcal{A}_{1}} \tilde{g}_{\mathcal{A}_{1}}\right. \\
& \left.+\left(\beta^{*} \alpha+|\beta|^{2}\right) g_{\mathcal{A}_{1}} \overline{\bar{g}}_{\mathcal{A}_{1}}\left|0_{\mathcal{A}_{1}}\right\rangle\left\langle 0_{\mathcal{A}_{1}}\right| \overline{\bar{g}}_{\mathcal{A}_{1}} g_{\mathcal{A}_{1}} \tilde{g}_{\mathcal{A}_{1}}\right] .
\end{aligned}
$$

In order to obtain the von Neumann entropy of subsystem $\mathcal{A}_{1}$ we begin by computing the second power of $\rho_{\mathcal{A}_{1}}$, 


$$
\begin{aligned}
\rho_{\mathcal{A}_{1}}^{2}=\frac{1}{\mathcal{N}^{2}} \sum_{g, g^{\prime} \in G_{\mathcal{A}}, \tilde{g}, \tilde{g}^{\prime} \in G_{\mathcal{A}_{1}}} & {\left[\left(|\alpha|^{2}+\alpha^{*} \beta\right)^{2} g_{\mathcal{A}_{1}} \bar{g}_{\mathcal{A}_{1}}\left|0_{\mathcal{A}_{1}}\right\rangle\left\langle 0_{\mathcal{A}_{1}}\left|g_{\mathcal{A}_{1}} \tilde{g}_{\mathcal{A}_{1}} g_{\mathcal{A}_{1}}^{\prime}\right| 0_{\mathcal{A}_{1}}\right\rangle\left\langle 0_{\mathcal{A}_{1}}\right| \bar{g}_{\mathcal{A}_{1}} g_{\mathcal{A}_{1}}^{\prime} \tilde{g}_{\mathcal{A}_{1}}^{\prime}\right.} \\
+ & +\left(|\alpha|^{2}+\alpha^{*} \beta\right)\left(\beta^{*} \alpha+|\beta|^{2}\right) g_{\mathcal{A}_{1}} \bar{g}_{\mathcal{A}_{1}}\left|0_{\mathcal{A}_{1}}\right\rangle\left\langle 0_{\mathcal{A}_{1}}\left|\bar{g}_{\mathcal{A}_{1}} g_{\mathcal{A}_{1}} \tilde{g}_{\mathcal{A}_{1}} g_{\mathcal{A}_{1}}^{\prime} \overline{\bar{g}}_{\mathcal{A}_{1}}\right| 0_{\mathcal{A}_{1}}\right\rangle\left\langle 0_{\mathcal{A}_{1}}\right| \overline{\bar{g}}_{\mathcal{A}_{1}} g_{\mathcal{A}_{1}}^{\prime} \tilde{g}_{\mathcal{A}_{1}}^{\prime} \\
& +\left(\beta^{*} \alpha+|\beta|^{2}\right)\left(|\alpha|^{2}+\alpha^{*} \beta\right) g_{\mathcal{A}_{1}} \overline{\bar{g}}_{\mathcal{A}_{1}}\left|0_{\mathcal{A}_{1}}\right\rangle\left\langle 0_{\mathcal{A}_{1}}\left|\overline{\bar{g}}_{\mathcal{A}_{1}} g_{\mathcal{A}_{1}} \tilde{g}_{\mathcal{A}_{1}} g_{\mathcal{A}_{1}}^{\prime} \bar{g}_{\mathcal{A}_{1}}\right| 0_{\mathcal{A}_{1}}\right\rangle\left\langle 0_{\mathcal{A}_{1}}\right| \bar{g}_{\mathcal{A}_{1}} g_{\mathcal{A}_{1}}^{\prime} \tilde{g}_{\mathcal{A}_{1}}^{\prime} \\
& \left.+\left(\beta^{*} \alpha+|\beta|^{2}\right)^{2} g_{\mathcal{A}_{1}} \overline{\bar{g}}_{\mathcal{A}_{1}}\left|0_{\mathcal{A}_{1}}\right\rangle\left\langle 0_{\mathcal{A}_{1}}\left|g_{\mathcal{A}_{1}} \tilde{g}_{\mathcal{A}_{1}} g_{\mathcal{A}_{1}}^{\prime}\right| 0_{\mathcal{A}_{1}}\right\rangle\left\langle 0_{\mathcal{A}_{1}}\right| \overline{\bar{g}}_{\mathcal{A}_{1}} g_{\mathcal{A}_{1}}^{\prime} \tilde{g}_{\mathcal{A}_{1}}^{\prime}\right]
\end{aligned}
$$

The first and last term involve the expectation value

$$
\left\langle 0_{\mathcal{A}_{1}}\left|g_{\mathcal{A}_{1}} \tilde{g}_{\mathcal{A}_{1}} g_{\mathcal{A}_{1}}^{\prime}\right| 0_{\mathcal{A}_{1}}\right\rangle,
$$

which can be evaluated with the help of the change of variable $g^{\prime} \rightarrow g \tilde{g} g^{\prime}$. This then imposes $g_{\mathcal{A}_{1}}^{\prime}=\mathbf{1}_{\mathcal{A}_{1}}$. The second and third terms involve instead the expectation value

$$
\left\langle 0_{\mathcal{A}_{1}}\left|\overline{\bar{g}}_{\mathcal{A}_{1}} g_{\mathcal{A}_{1}} \tilde{g}_{\mathcal{A}_{1}} g_{\mathcal{A}_{1}}^{\prime} \bar{g}_{\mathcal{A}_{1}}\right| 0_{\mathcal{A}_{1}}\right\rangle
$$

$G_{\mathcal{A}}$ exists such that $h_{\mathcal{A}_{1}}=\bar{g}_{\mathcal{A}_{1}} \overline{\bar{g}}_{\mathcal{A}_{1}}$. Our assumption of the existence of $g^{*} \in G_{\mathcal{A}}$ clearly satisfies this condition for $h=g^{*}$ (this is again sufficient but not necessary), and we can perform the change of variable $g^{\prime} \rightarrow g \tilde{g} g^{*} g^{\prime}$ whereby the expectation value reduces to $\left\langle 0_{\mathcal{A}_{1}}\left|g_{\mathcal{A}_{1}}^{\prime}\right| 0_{\mathcal{A}_{1}}\right\rangle$. In all cases, the dependence on $g^{\prime}$ disappears, and it can thus be trivially summed over, resulting in an overall factor of $\left|G_{\mathcal{A}_{2}}\right|$.

After these steps, we arrive at the expression,

which once again vanishes identically unless an element $h \in$

$$
\begin{aligned}
\rho_{\mathcal{A}_{1}}^{2}=\frac{\left|G_{\mathcal{A}_{2}}\right|}{\mathcal{N}^{2}} \sum_{g \in G_{\mathcal{A}}} \sum_{\tilde{g}, \tilde{g}^{\prime} \in G_{\mathcal{A}_{1}}} & {\left[\left(|\alpha|^{2}+\alpha^{*} \beta\right)^{2} g_{\mathcal{A}_{1}} \bar{g}_{\mathcal{A}_{1}}\left|0_{\mathcal{A}_{1}}\right\rangle\left\langle 0_{\mathcal{A}_{1}}\right| \bar{g}_{\mathcal{A}_{1}} g_{\mathcal{A}_{1}} \tilde{g}_{\mathcal{A}_{1}} \tilde{g}_{\mathcal{A}_{1}}^{\prime}\right.} \\
+ & \left(|\alpha|^{2}+\alpha^{*} \beta\right)\left(\beta^{*} \alpha+|\beta|^{2}\right) g_{\mathcal{A}_{1}} \bar{g}_{\mathcal{A}_{1}}\left|0_{\mathcal{A}_{1}}\right\rangle\left\langle 0_{\mathcal{A}_{1}}\right| \bar{g}_{\mathcal{A}_{1}} g_{\mathcal{A}_{1}} \tilde{g}_{\mathcal{A}_{1}} \tilde{g}_{\mathcal{A}_{1}}^{\prime} \\
& +\left(\beta^{*} \alpha+|\beta|^{2}\right)\left(|\alpha|^{2}+\alpha^{*} \beta\right) g_{\mathcal{A}_{1}} \overline{\bar{g}}_{\mathcal{A}_{1}}\left|0_{\mathcal{A}_{1}}\right\rangle\left\langle 0_{\mathcal{A}_{1}}\right| \overline{\bar{g}}_{\mathcal{A}_{1}} g_{\mathcal{A}_{1}} \tilde{g}_{\mathcal{A}_{1}} \tilde{g}_{\mathcal{A}_{1}}^{\prime} \\
& \left.+\left(\beta^{*} \alpha+|\beta|^{2}\right)^{2} g_{\mathcal{A}_{1}} \overline{\bar{g}}_{\mathcal{A}_{1}}\left|0_{\mathcal{A}_{1}}\right\rangle\left\langle 0_{\mathcal{A}_{1}}\right| \overline{\bar{g}}_{\mathcal{A}_{1}} g_{\mathcal{A}_{1}} \tilde{g}_{\mathcal{A}_{1}} \tilde{g}_{\mathcal{A}_{1}}^{\prime}\right]
\end{aligned}
$$

where we used the fact that $g_{\mathcal{A}_{1}}^{*} \bar{g}_{\mathcal{A}_{1}}=\overline{\bar{g}}_{\mathcal{A}_{1}}$ and $g_{\mathcal{A}_{1}}^{*} \overline{\bar{g}}_{\mathcal{A}_{1}}=$ $\bar{g}_{\mathcal{A}_{1}}$. We notice that $\tilde{g}$ and $\tilde{g}^{\prime}$ always appear as the product $\tilde{g} \tilde{g}^{\prime}$. A straightforward redefinition $\tilde{g} \rightarrow \tilde{g} \tilde{g}^{\prime}$ allows to remove the dependence on $\tilde{g}^{\prime}$, which thus resums to a factor $\left|G_{\mathcal{A}_{1}}\right|$. Moreover, the first two terms and the latter two terms differ from one another only by their coefficients in curved brackets, which can thus be combined to:

$$
\begin{gathered}
\left(|\alpha|^{2}+\alpha^{*} \beta\right)(1+x) \\
\left(\beta^{*} \alpha+|\beta|^{2}\right)(1+x),
\end{gathered}
$$

respectively, where we used the normalisation condition $|\alpha|^{2}+|\beta|^{2}=1$ and the shorthand notation introduced earlier, $x=\alpha^{*} \beta+\beta^{*} \alpha$. We finally recognise that

$$
\begin{aligned}
\rho_{\mathcal{A}_{1}}^{2}= & \frac{\left|G_{\mathcal{A}_{1}}\right|\left|G_{\mathcal{A}_{2}}\right|}{\mathcal{N}^{2}} \sum_{g \in G_{\mathcal{A}}} \sum_{\tilde{g} \in G_{\mathcal{A}_{1}}}(1+x) \\
\times & {\left[\left(|\alpha|^{2}+\alpha^{*} \beta\right) g_{\mathcal{A}_{1}} \bar{g}_{\mathcal{A}_{1}}\left|0_{\mathcal{A}_{1}}\right\rangle\left\langle 0_{\mathcal{A}_{1}}\right| \bar{g}_{\mathcal{A}_{1}} g_{\mathcal{A}_{1}} \tilde{g}_{\mathcal{A}_{1}}\right.} \\
& \left.+\left(\beta^{*} \alpha+|\beta|^{2}\right) g_{\mathcal{A}_{1}} \overline{\bar{g}}_{\mathcal{A}_{1}}\left|0_{\mathcal{A}_{1}}\right\rangle\left\langle 0_{\mathcal{A}_{1}}\right| \overline{\bar{g}}_{\mathcal{A}_{1}} g_{\mathcal{A}_{1}} \tilde{g}_{\mathcal{A}_{1}}\right] \\
= & \frac{\left|G_{\mathcal{A}_{1}}\right|\left|G_{\mathcal{A}_{2}}\right|}{\mathcal{N}}(1+x) \rho_{\mathcal{A}_{1}} \\
= & \frac{\left|G_{\mathcal{A}_{1}}\right|\left|G_{\mathcal{A}_{2}}\right|}{\left|G_{\mathcal{A}}\right|} \rho_{\mathcal{A}_{1}},
\end{aligned}
$$

which is the same as Eq. (13) in the main text.

To summarise, provided that the states in the superposition satisfy the condition $\bar{g}_{\mathcal{A}} \overline{\bar{g}}_{\mathcal{A}} \otimes \mathbf{1}_{\mathcal{B}} \in G$, the result obtained in the main text holds: the von Neumann entropy of $\mathcal{A}_{1}$ after subsystem $\mathcal{B}$ has been projected onto the superposition is a direct measure of the topological entropy of the system, devoid of any area law or other terms scaling with the size of the partitions. (We refrain here from verifying that the same is true for the entanglement negativity as well.)

What happens if the superposition does not satisfy this condition? We need to start again from Eq. (E5), where we now have $\mathcal{N}=\left|G_{\mathcal{A}}\right|$. In this case, the calculations involve significantly longer expressions and we do not report them here in full. Once again, we find that $\rho_{\mathcal{A}_{1}}$ depends on the expectation value

$$
\left\langle 0_{\mathcal{A}_{2}}\left|\overline{\bar{g}}_{\mathcal{A}_{2}} g_{\mathcal{A}_{2}}^{\prime} g_{\mathcal{A}_{2}} \bar{g}_{\mathcal{A}_{2}}\right| 0_{\mathcal{A}_{2}}\right\rangle
$$

and $\rho_{\mathcal{A}_{1}}^{2}$ further depends on the expectation value

$$
\left\langle 0_{\mathcal{A}_{1}}\left|\overline{\bar{g}}_{\mathcal{A}_{1}} g_{\mathcal{A}_{1}} \tilde{g}_{\mathcal{A}_{1}} g_{\mathcal{A}_{1}}^{\prime} \bar{g}_{\mathcal{A}_{1}}\right| 0_{\mathcal{A}_{1}}\right\rangle
$$

for $g, g^{\prime} \in G_{\mathcal{A}}$ and $\tilde{g} \in G_{\mathcal{A}_{1}}$. Depending on the nature of the elements $\bar{g}$ and $\overline{\bar{g}}$, one can show that all possible combinations are allowed, namely:

(i) both expectation values admit a non-vanishing solution; 
(ii) only one of them does; or

(iii) both vanish identically.

(We note that option (i) were both admit a solution does not contradict the assumption $\bar{g}_{\mathcal{A}} \overline{\bar{g}}_{\mathcal{A}} \otimes \mathbf{1}_{\mathcal{B}} \notin G$.)

Let us consider in detail scenario (iii) where both expectation values above vanish identically for all choices of $g, g^{\prime} \in$ $G_{\mathcal{A}}$ and $\tilde{g} \in G_{\mathcal{A}_{1}}$. As a result, the terms proportional to $\alpha^{*} \beta$ and to $\beta^{*} \alpha$ in the reduced density matrix $\rho_{\mathcal{A}_{1}}$ in Eq. (E11) are absent:

$$
\begin{aligned}
& \rho_{\mathcal{A}_{1}}= \frac{1}{\left|G_{\mathcal{A}}\right|} \sum_{g \in G_{\mathcal{A}}} \sum_{\tilde{g} \in G_{\mathcal{A}_{1}}} \\
& \times\left[|\alpha|^{2} g_{\mathcal{A}_{1}} \bar{g}_{\mathcal{A}_{1}}\left|0_{\mathcal{A}_{1}}\right\rangle\left\langle 0_{\mathcal{A}_{1}}\right| \bar{g}_{\mathcal{A}_{1}} g_{\mathcal{A}_{1}} \tilde{g}_{\mathcal{A}_{1}}\right. \\
&\left.\quad+|\beta|^{2} g_{\mathcal{A}_{1}} \overline{\bar{g}}_{\mathcal{A}_{1}}\left|0_{\mathcal{A}_{1}}\right\rangle\left\langle 0_{\mathcal{A}_{1}}\right| \overline{\bar{g}}_{\mathcal{A}_{1}} g_{\mathcal{A}_{1}} \tilde{g}_{\mathcal{A}_{1}}\right] .
\end{aligned}
$$

The calculation of its second power results in the same terms that were obtained in Eq. (E12), up to trivial changes in the constant coefficients in round brackets:

$$
\begin{aligned}
\rho_{\mathcal{A}_{1}}^{2} & =\frac{\left|G_{\mathcal{A}_{1}}\right|\left|G_{\mathcal{A}_{2}}\right|}{\left|G_{\mathcal{A}}\right|^{2}} \sum_{g \in G_{\mathcal{A}}} \sum_{\tilde{g} \in G_{\mathcal{A}_{1}}} \\
& \times\left[|\alpha|^{4} g_{\mathcal{A}_{1}} \bar{g}_{\mathcal{A}_{1}}\left|0_{\mathcal{A}_{1}}\right\rangle\left\langle 0_{\mathcal{A}_{1}}\right| \bar{g}_{\mathcal{A}_{1}} g_{\mathcal{A}_{1}} \tilde{g}_{\mathcal{A}_{1}}\right. \\
& \left.+|\beta|^{4} g_{\mathcal{A}_{1}} \overline{\bar{g}}_{\mathcal{A}_{1}}\left|0_{\mathcal{A}_{1}}\right\rangle\left\langle 0_{\mathcal{A}_{1}}\right| \overline{\bar{g}}_{\mathcal{A}_{1}} g_{\mathcal{A}_{1}} \tilde{g}_{\mathcal{A}_{1}}\right] .
\end{aligned}
$$

This result can be straightforwardly iterated to give

$$
\begin{aligned}
\rho_{\mathcal{A}_{1}}^{n} & =\frac{\left(\left|G_{\mathcal{A}_{1}}\right|\left|G_{\mathcal{A}_{2}}\right|\right)^{n-1}}{\left|G_{\mathcal{A}}\right|^{n}} \sum_{g \in G_{\mathcal{A}}} \sum_{\tilde{g} \in G_{\mathcal{A}_{1}}} \\
& \times\left[|\alpha|^{2 n} g_{\mathcal{A}_{1}} \bar{g}_{\mathcal{A}_{1}}\left|0_{\mathcal{A}_{1}}\right\rangle\left\langle 0_{\mathcal{A}_{1}}\right| \bar{g}_{\mathcal{A}_{1}} g_{\mathcal{A}_{1}} \tilde{g}_{\mathcal{A}_{1}}\right. \\
& \left.+|\beta|^{2 n} g_{\mathcal{A}_{1}} \overline{\bar{g}}_{\mathcal{A}_{1}}\left|0_{\mathcal{A}_{1}}\right\rangle\left\langle 0_{\mathcal{A}_{1}}\right| \overline{\bar{g}}_{\mathcal{A}_{1}} g_{\mathcal{A}_{1}} \tilde{g}_{\mathcal{A}_{1}}\right],
\end{aligned}
$$

and thus

$$
\operatorname{Tr}\left(\rho_{\mathcal{A}_{1}}^{n}\right)=\left(\frac{\left|G_{\mathcal{A}_{1}}\right|\left|G_{\mathcal{A}_{2}}\right|}{\left|G_{\mathcal{A}}\right|}\right)^{n-1}\left[|\alpha|^{2 n}+|\beta|^{2 n}\right],
$$

from which we obtain the von Neumann entropy:

$$
\begin{aligned}
S_{\mathrm{vN}}^{\left(\mathcal{A}_{1}\right)} & =-\lim _{n \rightarrow 1} \partial_{n}\left[\operatorname{Tr}\left(\rho_{\mathcal{A}_{1}}^{n}\right)\right] \\
& =\ln \frac{\left|G_{\mathcal{A}}\right|}{\left|G_{\mathcal{A}_{1}}\right|\left|G_{\mathcal{A}_{2}}\right|}-|\alpha|^{2} \ln |\alpha|^{2}-|\beta|^{2} \ln |\beta|^{2} .
\end{aligned}
$$

In addition to the usual topological contribution, in this case a new term appears that reminds of the classical entropy of mixing between the two states in the superposition (recall that $\left.|\alpha|^{2}+|\beta|^{2}=1\right)$.

Similarly, although the derivation is not reported here, one can compute the entanglement negativity in this case. After a few lines of algebra, one arrives at the expressions

$$
\begin{aligned}
\operatorname{Tr}\left[\left(\rho_{\mathcal{A}}^{T_{2}}\right)^{2 n}\right] & =\left(\frac{\left|G_{\mathcal{A}_{1}}\right|\left|G_{\mathcal{A}_{2}}\right|}{\left|G_{\mathcal{A}}\right|}\right)^{2 n-2} \\
& \times\left[|\alpha|^{2 n}+|\beta|^{2 n}\right]^{2} \\
\operatorname{Tr}\left[\left(\rho_{\mathcal{A}}^{T_{2}}\right)^{2 n+1}\right] & =\left(\frac{\left|G_{\mathcal{A}_{1}}\right|\left|G_{\mathcal{A}_{2}}\right|}{\left|G_{\mathcal{A}}\right|}\right)^{2 n} \\
& \times\left[|\alpha|^{2(2 n+1)}+|\beta|^{2(2 n+1)}\right] .
\end{aligned}
$$

We see once again that the even and odd series lead to different analytic continuations. On the one hand, the odd series for $(2 n+1) \rightarrow 1$ (i.e., $n \rightarrow 0)$ tends to 1 , as expected for $\operatorname{Tr}\left(\rho_{\mathcal{A}}^{T_{2}}\right)$. On the other hand, the even series for $2 n \rightarrow 1$ (i.e., $n \rightarrow 1 / 2)$ tends to

$$
\left\|\rho_{\mathcal{A}}^{T_{2}}\right\|_{1}=\frac{\left|G_{\mathcal{A}}\right|}{\left|G_{\mathcal{A}_{1}}\right|\left|G_{\mathcal{A}_{2}}\right|}(|\alpha|+|\beta|)^{2}
$$

and we obtain a finite entanglement negativity

$$
\begin{aligned}
\mathcal{E} & \equiv \ln \left\|\rho_{\mathcal{A}}^{T_{2}}\right\|_{1} \\
& =\ln \frac{\left|G_{\mathcal{A}}\right|}{\left|G_{\mathcal{A}_{1}}\right|\left|G_{\mathcal{A}_{2}}\right|}+2 \ln (|\alpha|+|\beta|) .
\end{aligned}
$$

Both the von Neumann entropy and the entanglement negativity carry an additional contribution determined by the coefficients in the superposition, on top of the expected topological contribution. It is interesting to notice that, although similar in features (e.g., both peak at $\ln 2$ when $|\alpha|=|\beta|=$ $1 / \sqrt{2}$ ), the two additional contributions are in fact not identical.

Regarding the two remaining cases, we find through similar calculations that the result in the main text holds for (ii), irrespective of which of the two expectation values vanishes and which does not. Case (i) appears to require significantly more elaborate calculations that were not carried out in this work, although we expect that the result in the main text holds also in this case.

In conclusion, we can understand the results in this appendix as follows. The existence of a non-trivial subgroup $G_{\mathcal{A}} \subset G$ (i.e., $\left|G_{\mathcal{A}}\right|>1$ ) tells us that the projection of $\mathcal{B}$ onto a given state does not fully determine the state of the system. Indeed, the elements of $G_{\mathcal{A}}$ label the states in the GS superposition that contribute to the projected density matrix.

Now, let us compare the projection of $\mathcal{B}$ onto two different states represented by $\bar{g}$ and $\overline{\bar{g}}$, and let us focus on the case at hand where we are interested in measuring the surviving entanglement between $\mathcal{A}_{1}$ and $\mathcal{A}_{2}$. So long as the remaining freedom in the system (i.e., the summation over elements of $\left.G_{\mathcal{A}}\right)$ allows to interchange $\bar{g}$ with $\overline{\bar{g}}$ on at least one of the two components of $\mathcal{A}$, then the two projected measures of entanglement are in fact one and the same. In mathematical terms, this translates into at least one of the following conditions being satisfied,

$$
\begin{aligned}
\exists h \in G_{\mathcal{A}} & : h_{\mathcal{A}_{2}} \bar{g}_{\mathcal{A}_{2}}=\overline{\bar{g}}_{\mathcal{A}_{2}} \\
\exists h^{\prime} \in G_{\mathcal{A}} & : h_{\mathcal{A}_{1}}^{\prime} \bar{g}_{\mathcal{A}_{1}}=\overline{\bar{g}}_{\mathcal{A}_{1}},
\end{aligned}
$$


which can in turn be expressed as the expectation values encountered above, Eq. (E21) and Eq. (E22):

$$
\begin{gathered}
\left\langle 0_{\mathcal{A}_{2}}\left|\overline{\bar{g}}_{\mathcal{A}_{2}} h_{\mathcal{A}_{2}} \bar{g}_{\mathcal{A}_{2}}\right| 0_{\mathcal{A}_{2}}\right\rangle \\
\left\langle 0_{\mathcal{A}_{1}}\left|\overline{\bar{g}}_{\mathcal{A}_{1}} h_{\mathcal{A}_{1}}^{\prime} \bar{g}_{\mathcal{A}_{1}}\right| 0_{\mathcal{A}_{1}}\right\rangle .
\end{gathered}
$$

for $h=g^{\prime} g$ and $h^{\prime}=g \tilde{g} g^{\prime}\left(g, g^{\prime} \in G_{\mathcal{A}}\right.$ and $\left.\tilde{g} \in G_{\mathcal{A}_{1}} \subset G_{\mathcal{A}}\right)$.

Whenever at least one of the two expectation values does not vanish (i.e., cases (i)-(ii) above), we are essentially free to interchange $\bar{g}$ and $\overline{\bar{g}}$ in the entanglement calculation and one expects the result to be the same as in the main text, where only one state was considered.

On the contrary, when both expectation values vanish identically, the two states $\bar{g}$ and $\overline{\bar{g}}$ give inherently different contributions and we find that, in addition to the expected topological entropy, the von Neumann entropy and entanglement negativity pick up a contribution dependent on the chosen quantum superposition of $\bar{g}$ and $\overline{\bar{g}}$. This happens, for instance, when $\bar{g}=\mathbf{1}$ and $\overline{\bar{g}}$ is given by the product of two star operators, one acting simultaneously on $\mathcal{A}_{1}$ and $\mathcal{B}_{1}$, and the other acting simultaneously on $\mathcal{A}_{2}$ and $\mathcal{B}_{2}$.
[1] A. Y. Kitaev and J. Preskill, Phys. Rev. Lett. 96, 110404 (2006).

[2] M. Levin and X.-G. Wen, Phys. Rev. Lett. 96, 110405 (2006).

[3] K. Zyczkowski, P. Horodecki,A. Sanpera, and M. Lewenstein, Phys. Rev. A 58, 883 (1998); see also K. Zyczkowski, Phys. Rev. A 60, 3496 (1999).

[4] J. Lee, M.S. Kim, Y.J. Park, and S. Lee, J. Mod. Opt. 47, 2151 (2000).

[5] J. Eisert, PhD thesis, University of Potsdam (2001).

[6] M. B. Plenio, Phys. Rev. Lett. 95, 090503 (2005). Albeit published at a later date than Ref. 4, it is the author's understanding that the work carried out by Eisert and Plenio, as well as that by Vidal and Werner, was in fact contemporary (and independent) of Lee et al. For a historical account see Ref. 16.

[7] G. Vidal and R. F. Werner, Phys. Rev. A 65, 032314 (2002).

[8] Y. A. Lee and G. Vidal, Phys. Rev. A 88, 042318 (2013).

[9] C. Castelnovo, Phys. Rev. A 88, 042319 (2013).

[10] J.-M. Stéphan, G. Misguich and V. Pasquier, J. Stat. Mech. P02003 (2012).

[11] A. Y. Kitaev, Ann. Phys. (N.Y.) 303, 2 (2003).

[12] A. Hamma, R. Ionicioiu, and P. Zanardi, Phys. Rev. A 71, 022315 (2005).

[13] C. Castelnovo and C. Chamon, Phys. Rev. B 77, 054433 (2008).
[14] C. Castelnovo and C. Chamon, Phys. Rev. B 76, 174416 (2007).

[15] P. Calabrese, J. Cardy, E. Tonni, Phys. Rev. Lett. 109, 130502 (2012).

[16] P. Calabrese, J. Cardy, E. Tonni, J. Stat. Mech. P02008 (2013); P. Calabrese, L. Tagliacozzo, and E. Tonni, J. Stat. Mech. P05002 (2013).

[17] V. Alba, J. Stat. Mech. P05013 (2013).

[18] B. Sutherland, J. Math. Phys. 11, 3183 (1970); C. Fan and F. Y. Wu, Phys. Rev. B 2, 723 (1970).

[19] C. Castelnovo and C. Chamon, Phys. Rev. B 76, 184442 (2007).

[20] C. Castelnovo and C. Chamon, Phys. Rev. B 78, 155120 (2008).

[21] E. K.-H. Lee, R. Schaffer, S. Bhattacharjee, and Y. B. Kim, Phys. Rev. B 89, 045117 (2014); S.-B. Lee, E. K.-H. Lee, A. Paramekanti, Y. B. Kim, arXiv:1309.7050 (2013).

[22] I. Kimchi, J. G. Analytis, A. Vishwanath, arXiv:1309.1171 (2013).

[23] J. Nasu, T. Kaji, K. Matsuura, M. Udagawa, Y. Motome, arXiv:1309.3068 (2013).

[24] E. Fradkin and J. E. Moore, Phys. Rev. Lett. 97, 050404 (2006); E. Fradkin, J. Phys. A: Math. Theor. 42, 504011 (2009).

[25] M. Oshikawa, arXiv:1007.3739 (2010). 